\title{
Numerical Optimization of Plate-Line Design for Enhanced Wake-Vortex Decay
}

\author{
Anton Stephan,,- Jürgen Schrall, \pm and Frank Holzäpfel \\ DLR, German Aerospace Center, 82234 Oberpfaffenhofen, Germany
}

DOI: $10.2514 / 1 . C 033973$

\begin{abstract}
The design of plate lines as a ground-mounted device for wake-vortex decay enhancement is investigated in this work. The most important design parameters, the aspect ratio and plate distance, are analyzed for the wake vortices generated by two aircraft: the $\mathrm{A340}$ as well as the $\mathrm{A380}$. Large-eddy-simulations are used to simulate the wake-vortex evolution in ground proximity for different parameter combinations. Fully rolled-up wake vortices are initialized using a Lamb-Oseen vortex model resembling the characteristics of the two aircraft. With the stochastic so-called kriging method, estimates of the performance and respective probabilistic envelopes are given for the design parameter region, spanned by the large-eddy-simulation. The vortex circulation averaged over the rapid decay phase is taken as the objective function. The large-eddy-simulation parameters are selected in the vicinity of the expected optimum. An optimal parameter combination can be localized in the A340 case, as well as in the A380 case. For both cases, statistical relevance is provided. Moreover, it can be deduced that the optimal parameters for the $\mathbf{A 3 8 0}$ are also well suited for smaller aircraft like an A340.
\end{abstract}

\section{Nomenclature}

ar $\quad=$ plate aspect ratio

$b_{\text {ref }} \quad=\quad$ initial vortex separation of A340, $\mathrm{m}$

$b_{0}$

$C_{D}$

$C_{R}$

$H$

$h$

$L$

pd

$Q_{1}, Q_{2}, c R$

$R$

$r_{c}$

$S, K$

$t$

$t_{0}$

$u_{i}$

$w_{i}$

$w_{0}$

$x, x_{i}$

$x, y, z$

Z

$z_{0}$

$\Gamma_{0}$

$\gamma$

$\epsilon_{k}$

$\lambda$

$\mu$

$\nu, \nu_{t}$

$\sigma$ initial vortex separation, $\mathrm{m}$

drag coefficient, $1 / \mathrm{m}$

covariogram

plate height, $\mathrm{m}$

lag

plate length, $m$

plate separation

kriging quality measures

residual component

vortex core radius

kriging model parameters

time, $s$

time unit, $\mathrm{s}$

velocity components, $\mathrm{m} / \mathrm{s}$

weights

initial vortex descent speed, $\mathrm{m} / \mathrm{s}$

coordinates in parameter space

spatial coordinates, $\mathrm{m}$

objective function

initial vortex height, $m$

initial vortex circulation, $\mathrm{m}^{2} / \mathrm{s}$

variogram

regression error

Lagrange parameter

- mean value

$=$ kinematic and turbulent viscosity, $\mathrm{m}^{2} / \mathrm{s}$

$=$ standard deviation
Presented as Paper 2016-3135 at the 8th AIAA Atmospheric and Space Environments Conference, Washington, D.C., 13-17 June 2016; received 14 April 2016; revision received 20 July 2016; accepted for publication 31 July 2016; published online 29 November 2016. Copyright @ 2016 by Anton Stephan (DLR). Published by the American Institute of Aeronautics and Astronautics, Inc., with permission. All requests for copying and permission to reprint should be submitted to CCC at www.copyright.com; employ the ISSN 0021-8669 (print) or 1533-3868 (online) to initiate your request. See also AIAA Rights and Permissions www.aiaa.org/randp.

*Research Scientist, Institut für Physik der Atmosphäre; Anton.Stephan @ dlr.de.

${ }^{\dagger}$ Master Student, Institut für Physik der Atmosphäre.

${ }^{\ddagger}$ Senior Scientist, Institut für Physik der Atmosphäre.

\section{Introduction}

$\mathbf{F}$ LYING aircraft generate a pair of counter-rotating and longlived wake vortices. The generated two-vortex system can persist for several minutes, possessing a high amount of kinetic energy, and thereby posing a potential hazard to following aircraft. In ground proximity, transport and decay of the wake vortices are mainly determined by the wind conditions and the interaction with the ground surface. To avoid wake-vortex encounters, regulatory separation distances between aircraft pairs have to be maintained based on the leading aircraft's weight, which limits the capacity at the airports. Therefore, the investigation of wake-vortex decay is an important issue in commercial aviation [1-3] . The probability of encountering wake vortices increases significantly during the final approach in ground proximity because the vortices may not descend below the glidepath to leave the flight corridor vertically. Instead, they rebound, due to the interaction with the ground surface [4]. Moreover, the advection by weak crosswinds may compensate the self-induced lateral vortex transport. Therefore, the clearance of the flight corridor, by descent and advection of the vortices, may be strongly restricted. Due to the low height of the aircraft above the ground, the ability of the pilot to counteract the vortex-induced rolling moment is also limited. Consequently, the incident reporting scheme, compiled by the British National Air Traffic Services, lists most encounters at flight altitudes below $300 \mathrm{ft}$ [5].

The airport capacity problem is "as strong as ever" [6] This was concluded by Eurocontrol in the 2013 summary report titled "Challenges of Growth" [6]. Several scenarios for the future European airport demands were analyzed in this report, realizing that there would be unaccommodated flights in each case. In the most likely scenario of regulated growth, around 1.9 million flights would not be accommodated, constituting approximately $12 \%$ of the demand in 2035. For an optimal airport capacity, the aircraft separation of two following aircraft should be as small as possible. Still, the passenger and aircraft safety has to be ensured. As an operational minimum aircraft separation, which is used by air traffic control, the minimum radar separation corresponding mostly to $2.5 \mathrm{n}$ miles is employed, not including wake-vortex separation. Wakevortex separations for arrivals were introduced by the International Civil Aviation Organization (ICAO) in the 1970s, distinguishing three different aircraft weight classes: small up to $7000 \mathrm{~kg}$; medium up to $136,000 \mathrm{~kg}$; and heavy for the $\mathrm{A} 380$, based on the maximum takeoff weights. The separation distances between the different weight-class configurations vary from 2.5 to $6 \mathrm{n}$ miles [1]. In rapidly growing air traffic, the ICAO launched an initiative to increase airport capacity, aiming (eventually) at dynamic pairwise aircraft separations. This meant that, depending on the local environmental 


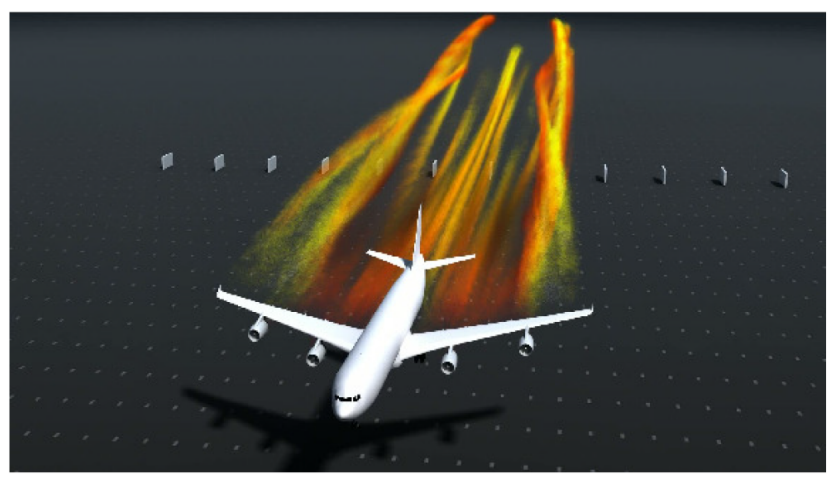

a) Wake-vortex rollup during final approach

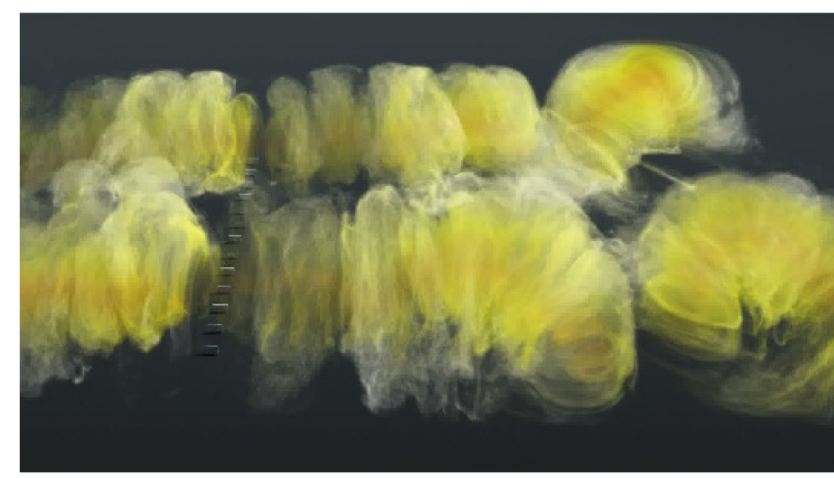

b) Vortex evolution after touchdown

Fig. 1 Hybrid RANS/LES simulation of aircraft landing with plate line.

conditions (like prevailing weather, runway configuration, and the aircraft pair), the separation was determined by a wake-vortex advisory system (WVAS). The Federal Aviation Administration, together with Eurocontrol, proposed a three-phase implementation program for the recategorization (the Wake Turbulence ReCategorization Program, or RECAT) of wake separation standards. The RECAT's phase 1 introduced six different static aircraft classes and extended the separation matrix. It was already successfully implemented at several U.S. airports, which included Memphis, Louisville, Cincinnati, and Atlanta [7]. This separation scheme was implemented at three French airports (Paris Charles de Gaulle, ParisLe Bourget, and Pontoise-Cormeilles-en-Vexin) in March 2016 [8]. Phase 2 aimed at static pairwise separations. Phase 3 shall establish dynamic pairwise separations of aircraft. Clearly, the ambitious goals of dynamic pairwise aircraft separations by RECAT will not be reached without an appropriate, optimally designed WVAS. Therefore, a deep understanding of the wake-vortex physics is essential, particularly for characterizing the processes in ground proximity.

In the case of crosswind speeds lower than the vortex descend speed, it may happen that the upwind vortex is not advected but located along the runway centerline [4]. A low turbulence intensity prevents the vortex from dissipating. Therefore, there is a strong need for devices in ground proximity for the destruction of wake vortices in meteorologically critical situations, or at least for weakening them to an uncritical level. There have been numerous attempts to accelerate vortex decay deliberately out of ground proximity [9]. However, much less work has been done on accelerating the wake-vortex decay in ground proximity. A device for active wake-vortex decay acceleration should be reasonably priced, safe, and effective, ideally permitting an aircraft separation reduction to radar separation. In that case, a WVAS should provide the necessary information that the glidepath is void of wake vortices. It should be particularly effective for heavy and superheavy aircraft.

Active and passive modifications at the aircraft have been tested in wind tunnels and towing tank facilities [9,10], in numerical simulations [11], and in real flight as part of the European project Aircraft Wing Advanced Technology Operations (AWIATOR). Early ideas to accelerate wake-vortex decay in ground proximity, such as applying jet blast and using water fountains or trees, have not been pursued further. Respective patents have been filed by F. Bao $\underline{\S}$ and A. Schröder and R. Konrath. Methods for wake-vortex decay enhancement can be divided into passive or active methods, as well as airborne or ground based. In projects funded by the European Commission (such as the Project on Wake-Vortex Characterization and Control, the Fundamental Research on Aircraft Wake Phenomena Project, and the Aircraft Wing Advanced Technology Operations Project), methods have been presented that modified the wing loading to trigger inherent vortex instabilities $[10,11]$. The development of suitable devices requires comprehensive theoretical,

${ }^{\S}$ Patent number DE 102005025004 A1 (2005).

qPatent number DE 102008054107 B3 (2008). numerical, and experimental efforts to prove the efficiency of the methodology. Suitable methods shall not increase drag and fuel consumption during cruise. In addition, they shall not decrease flight in any possible way. The airborne methods aim at triggering intrinsic instabilities like crow or crouch instabilities to accelerate the natural decay process. Several passive modifications of the wing configuration were proposed in [10,12], as well as active ones [13]. Special four-vortex system topologies to trigger instabilities were studied numerically [14].

A promising method to accelerate vortex decay, exploiting the fundamental properties of vortex dynamics, was presented in [15]. In this method, decay acceleration was achieved by introducing obstacles at the ground to trigger the turbulent interaction of primary and secondary vortices. This approach fell into the category of a passive ground-based method. A theoretical investigation of the interaction of antiparallel vortices of different strengths was performed [16]. Vortex decay could be initiated locally and accelerated in the vicinity of dedicated obstacles like plate lines installed at the ground; see Fig. 1. To put it simply, the obstacle caused the flow to redirect the force that normally caused the wake vortices above the obstacle to rebound into early turbulent vortex decay. It was found that a plate line might enforce the wake-vortex decay by more than $20 \%$ of the initial circulation at vortex ages in the order of wake-vortex separation times. The suggested ground-based and passive method required a relatively small technical effort to be tested and introduced at airports. A patent entitled "Surface Structure on a Ground Surface for Accelerating Decay of Wake Turbulence in the Short Final of an Approach to a Runway" was filed by Frank Holzäpfel-* that aimed at wake-vortex decay enhancement. The present work elaborates on a plate-line design for optimal vortex decay enhancement [15] using two methods: socalled temporal large-eddy simulations (LESs) and kriging interpolation.

After the wake-vortex measurement campaign at Oberpfaffenhofen Airport [17], the plate-line concept developed from the basic idea and the physical mechanisms behind it to a system that was intended for operational use. In the framework of the Single European Sky Air Traffic Management Research 2020 Project, a long-term study is planned at Vienna Airport to collect accurate statistics. The operational use of plate lines requires detailed analysis of the plateline parameters for an optimal effectiveness. Other institutions like the Nanyang Technological University of Singapore have also worked on plate-line design optimization, aiming at effective operational usage at the Changi Airport Singapore [18].

Finding the optimal plate-line design is a classical engineering design problem. Most engineering design problems require experiments and/or simulations to evaluate design objective and constraint functions as functions of design variables. Simulationdriven aerodynamic design is especially faced with large simulations that require much computation time [19]. Since few simulations can be performed due to computational constraints, surrogate models are used to fill the gap. In this paper, so-called kriging used in

\footnotetext{
**Patent number DE 102011010147 (2011).
} 
approximation-based surrogate models is applied to the design problem. Kriging (or Gaussian process regression) was developed by the French mathematician Georges Matheron [20]. This interpolation method stemmed from geostatistics and was based on the work of the South African geologist Danie Krige [21]. Originally, Krige was looking for the most likely distance-weighted gold distribution based on a few boreholes at the Witwatersrand reef complex in South Africa. Since then, kriging has been used in other disciplines besides geostatistics, such as for black-box modeling in computer experiments such as design studies, where costly simulations limit the number of experiments [19]. Stochastic processes are used as qualified estimator for the objective function. The use of a stochastic process in global optimization is called "Bayesian global optimization."

\section{Qualitative Discussion: Aims of the Study}

During the last decade, the evolution of wake vortices close to the ground has received much attention. In ground proximity, the vortices can persist for a long period and pose a hazard to the following aircraft $[4,22,23]$. The evolution of a wake-vortex system in ground proximity results in a complex three-dimensional flow; see Fig. 1. When counter-rotating vortices approach the ground or are generated at low altitudes, the proximity of a flat surface causes a divergence of the vortices. Induced by the vortices, an outboard directed flow on the surface establishes and vorticity of the opposite sign is produced in a boundary layer [24]. The induced flow near the surface experiences an adverse pressure gradient when passing the vortex cores, which is strong enough to cause a flow separation, leading to the formation of a separation bubble at the ground. Flow simulations have shown how pairs of secondary vortices are produced from the separation region [25-27]. The secondary vortices detach and interact with the primary vortices. The generation of secondary vorticity depends on the Reynolds number [23].

Recent technical developments that allow us to simulate the entire vortex evolution with a hybrid Reynolds-averaged Navier-Stokes (RANS)/LES approach, including the rollup phase as well as the decay phase, have revealed valuable insights for en route flight [28] as well as in ground proximity during landing with plate lines [29]. Figure 1 shows the rollup and vortex evolution in ground proximity subjected to a plate line. A tracer is seeded at isosurfaces of the vorticity magnitude, and the velocity of the tracer particles is shown with high velocities and low velocities. As a result, from [29], we know that the rollup process of the strongest vortices in the wake, the wingtip, and flap tip vortex proceeds very fast, especially in the vicinity of the ground. The topology of the vortices is much more complex than described in previous studies, particularly close to the touch down zone. However, in the proximity of the plate-line, vortices remain approximately straight. As the hybrid RANS/LES method requires much more computational as well as technical effort, we restrict ourselves to fully rolled-up vortices as a suitable approximation for the given parameter study.

The present study is focused on the numerical optimization of the plate-line design and deployment in terms of plate separation $p d$ as well as the aspect ratio $a r=L / H$ of the plates, where $L$ denotes the plate length and $H$ denotes the plate height; see Fig. 2. Here, we would like to discuss the role of these parameters qualitatively, based on vortex physics described in earlier work. From [15], it is known that higher plates favor wake-vortex decay, as the secondary vortex structures separating from the plates detach earlier, shifting the interaction of primary and secondary vortex structures to earlier times. Hence, higher plates favor the decay-enhancing effect. Here, we are limited by obstacle clearance criteria; consequently, the plate height does not require further investigation in our context. However, the optimal length of the plates for a fixed height is unknown. Keeping the plate height fixed, the variation of the aspect ratio implies a variable plate length with limits of zero and infinity. As elaborated in [15], the effectiveness of the plate line depends on vortex physics, particularly the behavior of the $\Omega$-shaped loop detaching from the plate. Two main vortex physics effects influence the flow (see also Sec. V):

1) Both branches of the $\Omega$ vortex induce a velocity of the vortex toward the primary vortex center.

2) The wrapped branches behave similar to a vortex ring and propagate axially along the primary vortex.

The plate-line length defines the width of the $\Omega$ vortex. The selfinduced velocity vanishes as the plate-line length approaches infinity. On the other hand, longer plate lines lead to much secondary vorticity, which starts to interact with the primary vortices at an early time. This favors the decay enhancement. We expect that both effects compete in a way that a natural optimum exists. One of the results of the current study is that this expectation is only partially met.

Also from a previous study [15], it is known that a massive continuous obstacle with a rectangular cross section is less effective than a line of plates, increasing the obstacle roughness. Obviously, the effect will decrease with a very large plate distance. This gives rise to a maximum effect for an optimal plate distance.

Varying both parameters (plate-line distance and aspect ratio of the plates), we wish to find optimum values to maximize the effect. This study is performed for two different aircraft types: the A340, as well as the $\mathrm{A} 380$.

As the simulations in this study will show the circulation curves for different configurations are crossing, so the circulation at a fixed vortex age is not exact enough; hence, it is not a suitable measure to evaluate the plate-line effect. Therefore, other metrics have to be found. Additionally, the effect of different aircraft sizes has to be

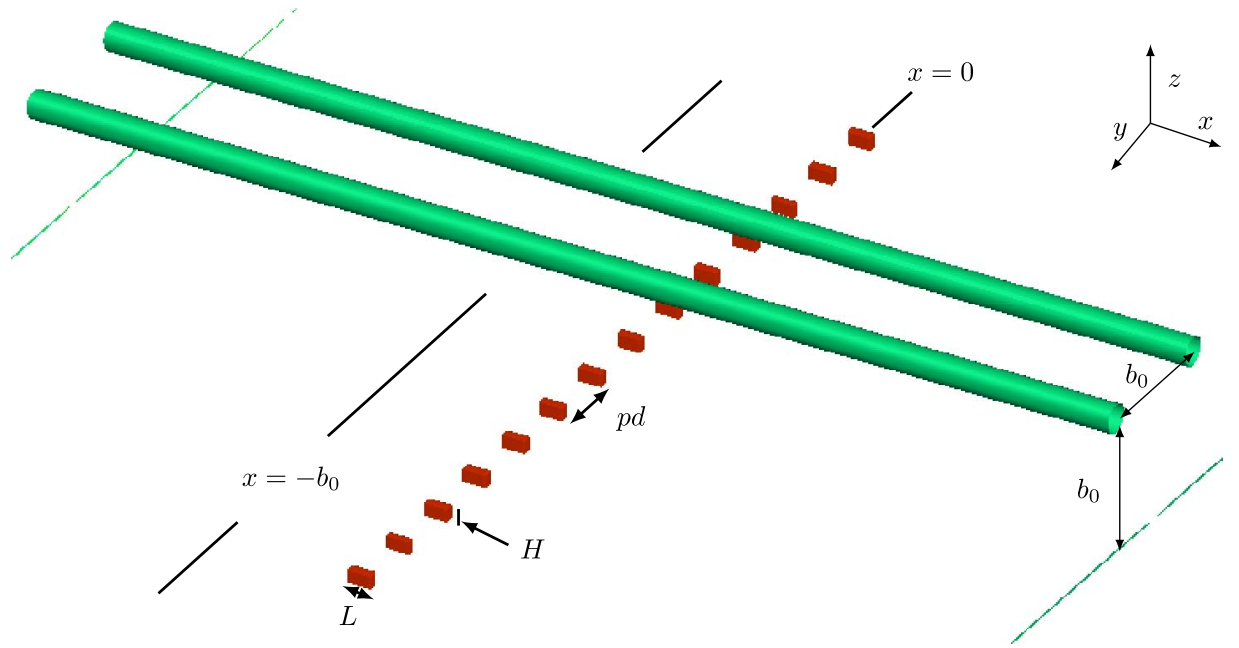

Fig. 2 Schematic of computational domain, initial vortex position, plate-line separation, height, and length. 
included in the study. Wake-vortex decay with plate lines presents a highly complex flow, with flow separation and vortex reconnection. The numerical method of choice is the LES. Hence, we are dealing with a nonlinear, unknown, cost-expensive black-box function, as described in [30]. The global optimization is a difficult task. The number of possible simulations is strongly limited. Here, we employ a Gaussian process regression approach called kriging for the interpolation of the objective function in the entire multiparameter space of interest. This finally yields optimal parameters and values for the objective function. In addition, the Gaussian process regression provides probability estimates of the interpolation in terms of the standard deviation, as well as upper and lower bounds.

\section{Numerical Method}

\section{A. Large-Eddy Simulation}

The interaction of a counter-rotating two-vortex system with a flat surface, using numerical simulations, has been investigated so far with different approaches, which either resolve or model the wall, i.e., wall-resolved direct numerical simulations (DNSs) [31] or LESs [32]. However, the resolution requirements for the boundary-layer flow limit the Reynolds number, not only in DNSs but also in LESs. In LESs, the subgrid-scale model only works properly if the modeled part of the flow contributes to a relatively small extent to the total turbulent kinetic energy (TKE). Close to walls, small structures mainly account for the TKE, such that the resolution close to walls has to be refined in LESs as well as in DNSs. Up to now, values of $R e_{\Gamma}=\Gamma / \nu$ on the order of 20,000 have been realized for wallresolved LESs for vortex flow [32]. This approach allows the investigation of coherent structures close to the wall at the expense of a relatively low $R e_{\Gamma}$.

The LES is performed using the incompressible Navier-Stokes code MGLET, which was developed at Technische Universität München, for solving the Navier-Stokes equations and the continuity equation [33]:

$$
\begin{gathered}
\frac{\partial u_{i}}{\partial t}+\frac{\partial\left(u_{i} u_{j}\right)}{\partial x_{j}}=-\frac{1}{\rho} \frac{\partial p^{\prime}}{\partial x_{i}}+\frac{\partial}{\partial x_{j}}\left(\left(\nu+\nu_{t}\right) 2 S_{i j}\right)-F_{D, i} \\
\frac{\partial u_{j}}{\partial x_{j}}=0
\end{gathered}
$$

Here, $u_{i}$ represents the velocity components in three spatial directions $(i=1,2$, or 3$), S_{i j}=\left(\partial u_{i} / \partial x_{j}+\partial u_{j} / \partial x_{i}\right) / 2$ denotes the strain rate tensor, and $p^{\prime}=p-p_{0}$ equals the pressure deviation from the reference state $p_{0}$. The kinematic viscosity is given as the sum of the molecular viscosity $\nu$ and eddy viscosity $\nu_{t}$ determined by means of a Lagrangian dynamic subgrid-scale model [34]. Equations (1) and (2) are solved by a finite volume approach using a fourth-order finite volume compact scheme [35]. A split-interface algorithm is used for the parallelization of the tridiagonal system [36], computing coefficients of the compact scheme. A third-order RungeKutta method is used for time integration. The simulations are performed in parallel, using a domain decomposition approach. Figure 2 displays the arrangement of the plates in a line perpendicular to the runway. The plate line is modeled by introducing a drag force source term, $-F_{D, i}=-C_{D}|u| u_{i}$, to Eq. (1) with a large drag coefficient at the plate [37]. Because wall-resolved LESs are performed, no ground wall model is used.

\section{B. Semivariogram}

A suitable way to interpolate experimental data $Z\left(\boldsymbol{x}_{i}\right)$ is kriging. As explained theoretically in the Appendix, the interpolation depends on a model for the variogram $\gamma$. A variogram $\gamma(h)$ as a function of the covariogram $C_{R}(h)$ has to be chosen, corresponding to [38]. Also called experimental variogram, this is a major part of geostatistics in general and kriging in particular.

For our simulations, $\boldsymbol{x}$ is a two-dimensional vector containing the parameters undergoing the parameter variation $\boldsymbol{x}=(p d, a r)$, where $p d$ denotes the distance between individual plates and $a r$ is the aspect ratio of a plate:

$$
a r=\frac{\text { plate height }(\text { fixed })}{\text { plate width }}
$$

The distance $h_{i j}$ is a measure for the degree of variation from each pair of points $\left(\boldsymbol{x}_{i}, \boldsymbol{x}_{j}\right)$. Each measurement $Z\left(\boldsymbol{x}_{i}\right), i=1, \ldots, n$ results from one simulation run with the varied parameters.

From $n$ measurements $Z\left(x_{1}\right), \ldots, Z\left(x_{n}\right)$, we get the raw variogram by plotting the square difference $(1 / 2)\left[Z\left(x_{i}\right)-Z\left(x_{j}\right)\right]^{2}$ against their separation $h_{i j}=\left\|x_{i}-x_{j}\right\|$ for all measurement pairs $(i \neq j),\|\cdot\|$. This results in a scatter plot of $(n(n-1) / 2)$ points for $n$ measurements, as performed in Sec. V.D.

The experimental variogram is a smooth line through this scatter plot. To achieve it, first, the separation distance is divided into $k$ consecutive intervals $\left[h_{k}^{l}, h_{k}^{u}\right]$, with each containing $N_{k}$ pairs of measurements $\left[Z\left(\boldsymbol{x}_{j}\right), Z\left(\boldsymbol{x}_{i}\right)\right]$. Next, the square difference of each individual lag is computed:

$$
\hat{\gamma}\left(h_{k}\right)=\frac{1}{2 N_{k}} \sum_{i=1}^{N_{k}}\left[Z\left(\boldsymbol{x}_{i}\right)-Z\left(\boldsymbol{x}_{j}\right)\right]^{2}
$$

indices $i, j$ refer to each pair of measurements $Z\left(\boldsymbol{x}_{i}\right)$ and $Z\left(\boldsymbol{x}_{j}\right)$ with

$$
h_{k}^{l} \leq\left\|x_{i}-x_{j}\right\|<h_{k}^{u}
$$

This interval is then averaged to obtain each single point of a lag

$$
h_{k}=\frac{1}{N_{k}} \sum_{i=1}^{N_{k}}\left\|\boldsymbol{x}_{i}-\boldsymbol{x}_{j}\right\|
$$

Connecting these points $\left(h_{k}, \hat{\gamma}\left(h_{k}\right)\right)$ leads to the experimental variogram.

One of the simplest models for the mean and the covariance function is given by

$$
E[Z(x)]=m
$$

and

$$
E\left[(Z(x)-m)\left(Z\left(x^{\prime}\right)-m\right)\right]=C_{R}(h)
$$

where

$$
h=\left\|\boldsymbol{x}-\boldsymbol{x}^{\prime}\right\|=\sqrt{\left(x_{1}-x^{\prime}{ }_{1}\right)^{2}+\left(x_{2}-x^{\prime}{ }_{2}\right)^{2}}
$$

implying that the mean is constant and the covariance function is only dependent on the distance between two points.

One of the common models recommended in $[39,40]$ is the Gaussian model. It defines the variogram and covariance function as

$$
\begin{gathered}
\gamma(h)=S \exp \left(-\frac{h^{2}}{K^{2}}\right) \\
C_{R}(h)=S\left(1-\exp \left(-\frac{h^{2}}{K^{2}}\right)\right)
\end{gathered}
$$

This method enables us to interpret the results of the LES and estimate the probability of a certain value being the optimum.

\section{Quality Measures of Kriging}

To quantify the fit of the kriging model, we examine the following statistics [38]: 


$$
Q_{1}=\frac{1}{n-1} \sum_{k=2}^{n} \epsilon_{k}
$$

with $\epsilon_{k}$ being the orthonormal residuals

$$
\epsilon_{k}=\frac{Z\left(x_{k}\right)-\hat{Z}_{k}}{\sigma_{k}}, \quad k=2, \ldots, n
$$

Here, $x_{k}$ represent a fixed progression of simulation points, and $\hat{Z}_{k}$ is the estimate at the $k$-th position using the points $x_{1}, \ldots, x_{k-1}$ :

$$
\hat{Z}_{k}=\sum_{i=1}^{k-1} w_{i} Z\left(x_{i}\right)
$$

The errors' mean $Q_{1}$ is a normal distributed random variable with $E\left[Q_{1}\right]=0$ and $E\left[Q_{1}^{2}\right]=(1 / n-1)$. A kriging model can be approved if

$$
\left|Q_{1}\right|<\frac{2}{\sqrt{n-1}}
$$

and can be considered safe. This rule involves a $5 \%$ probability to reject the correct model [38].

Another useful statistic is the errors' variance

$$
Q_{2}=\frac{1}{n-1} \sum_{k=2}^{n} \epsilon_{k}^{2}
$$

$Q_{2}$ is a random variable with mean $E\left[Q_{2}\right]=1$ and a variance that can be calculated for normally distributed $Z$ :

$$
E\left[\left(Q_{2}-1\right)^{2}\right]=(2 / n-1)
$$

The kriging model is of good quality if

$$
Q_{2} \in\left[1-\frac{2 \sqrt{2}}{\sqrt{n-1}}, 1+\frac{2 \sqrt{2}}{\sqrt{n-1}}\right]
$$

Additionally, a good index of the agreement between the model and the data is the stabilized geometric mean of the square residuals, given by

$$
c R=Q_{2} \exp \left(\frac{1}{n-1} \sum_{i=2}^{n} \ln \left(\sigma_{i}^{2}\right)\right)
$$

So, a good kriging model should result in being 1) $Q_{1}$ close to zero, 2) $Q_{2}$ close to one, and 3) $c R$ as small as possible.

\section{Simulations}

\section{A. Initial Vortex Pair}

We perform two sets of simulations for an A340 aircraft as well as for an A340 aircraft. Additional work, investigating even smaller aircraft types like the Gulfstream 550, can be found in [41]. For the A340, an initial circulation of $\Gamma_{0}=435 \mathrm{~m}^{2} / \mathrm{s}$ and an initial vortex separation of $b_{0}=b_{\text {ref }}=47.4 \mathrm{~m}$ are employed, leading to a vortex descend speed of $w_{0}=\Gamma_{0} / 2 \pi b_{0}=1.46 \mathrm{~m} / \mathrm{s}$ and a characteristic time of $t_{0}=b_{0} / w_{0}=32.5 \mathrm{~s}$. For the A380, an initial circulation of $\Gamma_{0}=820 \mathrm{~m}^{2} / \mathrm{s}$ and an initial vortex distance of $b_{0}=62.6 \mathrm{~m}$ are employed, leading to $w_{0}=2.1 \mathrm{~m} / \mathrm{s}$ and $t_{0}=29.7 \mathrm{~s}$, respectively. The vortices are initialized at a height of $b_{0}$, respectively, i.e., $z_{0}=47.4$ for the A340 and $z_{0}=62.6$ for the A380; see Fig. 2. The wake-vortex parameters are shown in Table 1 . Mirror vortices at the boundaries have been employed for initialization [15]. In agreement with previous work, the core radius is chosen to be $r_{c}=3 \mathrm{~m}$ in the A340 case, as well as in the A380 case. The mesh resolution of $0.5 \mathrm{~m}$ corresponds to previous work $[15,32]$. Note that another possibility is to normalize the initial setup and leave the circulation fixed while
Table 1 Wake-vortex parameters for different aircraft types

\begin{tabular}{lccccccc}
\hline \hline Index $k$ & Aircraft & $\Gamma_{0}, \mathrm{~m}^{2} / \mathrm{s}$ & $b_{0}, \mathrm{~m}$ & $t_{0}, \mathrm{~s}$ & $w_{0}, \mathrm{~m} / \mathrm{s}$ & $z_{0}, \mathrm{~m}$ & $R e_{\Gamma}$ \\
\hline A340 & $\mathrm{A} 340-300$ & 435 & 47.4 & 32.5 & 1.46 & 47.4 & 23,513 \\
A380 & A380-800 & 825 & 62.6 & 29.8 & 2.10 & 47.4 & 44,595 \\
\hline \hline
\end{tabular}

Table 2 Domain parameters, flight direction, width, height, and time

\begin{tabular}{lccccc}
\hline \hline Direction & Length & Size & Points & Total points & Cores \\
\hline$x$ & $384 \mathrm{~m}$ & $0.5 \mathrm{~m}$ & 768 & $157 \cdot 10^{6}$ & 2048 \\
$y$ & $320 \mathrm{~m}$ & $0.5 \mathrm{~m}$ & 640 & $157 \cdot 10^{6}$ & 2048 \\
$z$ & $128 \mathrm{~m}$ & $(0.1-0.5) \mathrm{m}$ & 320 & $157 \cdot 10^{6}$ & 2048 \\
$t$ & $97.5 \mathrm{~s}$ & $0.01 \mathrm{~s}$ & 9750 & $157 \cdot 10^{6}$ & 2048 \\
\hline \hline
\end{tabular}

varying plate distance, height, and length. However, by fixing plate height, the presentation of the results gets more vivid staying with actual dimensions.

\section{B. Computational Domain}

We used a similar domain for computation of the temporal largeeddy simulation, as in previous work $[15, \underline{32}]$. The mesh expressed in initial vortex separations of the $\mathrm{A} 340$ is approximately $8 b_{\text {ref }} \times 6.5 b_{\text {ref }} \times 3 b_{\text {ref }}$, cf. Fig. 2. The detailed mesh parameters can be found in Table 2 . At the front, rear, left, and right boundaries, we used periodic boundary condition. We applied a no-slip condition at the ground and a free slip condition at the top. The mesh was horizontally equidistant. The $x$ dimension was chosen such that the plate-line induced disturbance interacted with the boundary after $60 \mathrm{~s}$ or later in all simulated cases, which was the time period we were focusing at.

Since we are interested in the effects of the plate line near the ground, the LESs have to be wall-resolved. In the vertical direction, the mesh is stretched geometrically up to a height of $32 \mathrm{~m}$ and then is continued equidistantly until the top of the domain. Our LESs are computed with moderately high Reynolds numbers, $R e_{\Gamma}=\left(\Gamma_{0} / \nu\right)$; we have to set the molecular viscosity to $\nu=1.85 \cdot 10^{-2} \mathrm{~m}^{2} / \mathrm{s}$ to achieve Reynolds numbers on the order of $10^{5}$, depending on the simulated aircraft; see Table 1 . We reach our highest Reynolds numbers for the A380 with $\operatorname{Re}_{\Gamma}=44,595$, making that fine grid necessary.

In [42], resolution requirements of the flow over an airfoil, examined for nonequidistant grids, a number of $N \propto \operatorname{Re}_{\Gamma}^{1.8}$ grid points is recommended. This would be $\operatorname{Re}_{\Gamma}^{1.8} \approx 233 \cdot 10^{6}$ for this case and wall-resolved LESs. However, due to the vertical geometrically stretched grid at the ground, where resolution is most important, we are able to use only about two-thirds of the points, and therefore save computational time without using a wall model.

\section{Parameter Variation}

The characteristic parameters of our simulations are (see Fig. 2) as follows:

1) The first parameter is aircraft type. Wake vortices are generated according to the weight, wingspan, and speed of an aircraft.

2) The second parameter is the plate aspect ratio, which is the height-to-width ratio of each plate, where the plate height for the A340 as well as the A380 is fixed at $0.1 \cdot b_{\text {ref }}=4.74 \mathrm{~m}$. An initial aspect ratio of $a r=0.5$ corresponds to a $9.48 \mathrm{~m}$ width. A smaller aspect ratio of, e.g., $a r=0.25$ refers to a plate width of approximately $19 \mathrm{~m}$.

3) The third parameter is the plate distance. The distance between distinct plates of the obstacle line is an operationally important parameter.

Starting from a reference simulation with $a r=0.5$ and $p d=0.5 b_{\text {ref }}$, we perform variations of $a r=(0.25,0.7,1.0)$ and $p d / b_{\text {ref }}=(0.25,0.375,0.75,1.0)$. This leads to the resulting simulations listed in Table $\underline{3}$. 
Table 3 Performed simulations and parameters of numerical setup

\begin{tabular}{lcccc}
\hline \hline Case & Name & Aircraft & ar & pd \\
\hline 1,2 & Reference & A340, A380 & 0.50 & 0.50 \\
3,4 & Distance variation & A340, A380 & 0.50 & 0.25 \\
5,6 & Distance variation & A340, A380 & 0.50 & 0.375 \\
7,8 & Distance variation & A340, A380 & 0.50 & 0.75 \\
9,10 & Distance variation & A340, A380 & 0.50 & 1.00 \\
11,12 & Ratio variation & A340, A380 & 0.25 & 0.5 \\
13,14 & Ratio variation & A340, A380 & 0.7 & 0.5 \\
15,16 & Ratio variation & A340, A380 & 1.0 & 0.5 \\
17,18 & Mixed variation & A340, A380 & 0.7 & 0.75 \\
\hline \hline
\end{tabular}

\section{Results}

\section{A. Plate Distance}

The vortex circulation evolutions of that parameter study are depicted in Fig. 3; the circulation is plotted against time. For different plate distances, directly above the obstacle as well as in a characteristic distance of one initial vortex separation $b_{0}$, circulation is evaluated in slices perpendicular to the vortex axis. Figure 3 (left) shows the circulation of the A340, and Fig. 3 (right) shows the circulation of the A380. We observe for the A340 that, in the cases of $p d=\{0.25,0.375,0.5\}$, the phase of rapid decay (PRD) sets in around $t=30 \mathrm{~s}$, directly above the plate line. Analyzing the flowfields (Figs. 4 and 5), we observe the decay mechanism that was extensively described in [15]. In Figs. 4 and 5, an isosurface of the vorticity magnitude is displayed, which is shown with the vorticity in the $y$ direction. This gives a qualitative impression of the vortex physics behind the plate-line effect. Vortices can be distinguished, and the sense of rotation of the secondary vortices is shown. Secondary vortex structures (SVSs) develop above the obstacle; see Figs. $\underline{4 a}, \underline{4 c}$, and $4 \mathrm{e}$. They are stretched in the flowfield of the primary vortex and actively approach the vortex core. The evolving omega loop wraps around the primary vortex and induces helical disturbances propagating in the axial direction; see Figs. $\underline{4 \mathrm{~b}}, \underline{4 \mathrm{~d}}$, and 4f. This interaction drives the accelerated vortex decay.

The decay process is initiated above the obstacle and propagates in the axial direction. In the flowfield (see Figs. $4 \mathrm{a}$ and $4 \mathrm{c}$ ), the secondary vortex above the plate line has reached a mature state. For $p d=\{0.75,1.0\}$, the PRD sets in at approximately $t=40 \mathrm{~s}$. From Fig. $4 \mathrm{e}$, we observe that the secondary vortex loop needs more time to develop. The developing disturbance propagates axially in either direction (see Figs. $4 \mathrm{~b}$ and $4 \mathrm{~d}$ ), triggering the PRD in a distance of $b_{0}$ approximately $\overline{10} \mathrm{~s}$ later; see Fig. $\underline{3}$ (left). This exactly corresponds to the propagation time of the vortex helix. For $p d=\{0.75,1.0\}$, the entire decay process seems to be similar; however, it is shifted in time. The primary vortices are affected by the plates later; they descend in the intermediate spaces and are disturbed by the plates only after advection. Especially in the case of $p d=1.0$, we see a weaker decay.

In the case of an A380, we observe a similar behavior. For $p d=\{0.25,0.375,0.5,0.75\}$, we have mature secondary vortex loops and an early PRD at $t=30 \mathrm{~s}$; see Figs. $5 \mathrm{a}-$ dd and Fig. 3 (right). For $p d=1.0$, the decay process is strongly shifted in time: again, by approximately $10 \mathrm{~s}$. Due to larger vortices, it is just the $p d=1.0$ case where a shifted PRD is observed; $p d=0.75$ seems to still be sufficient for the primary vortices to hit the plates. Hence, from this analysis, we can separate roughly two decay regimes, depending on the maturity of the secondary vortex loop and whether plates are hit. However, it is unclear from Fig. 3 which parameter is optimal. Note that the rate of decay is similar in the case of a mature secondary vortex, proving that the main mechanism proceeds with minor deviations. Note also that, in the reference case, we observe a reorganization of the vortex strength above the plate line, at around $35 \mathrm{~s}$ (see Fig. 3 ) in contrast to the other cases. Although this effect has been observed in towing tank experiments, we do not have an explanation for the physical process behind this effect at this time [43].

The vortex trajectories coincide in all cases until approximately $t=30 \mathrm{~s}$; see Fig. $\underline{6}$. Interestingly, we observe an even deeper vortex descent, where vortices hit the plates, see the A340 case, $p d=\{0.25,0.375,0.5\}$. The rebound is weaker in this case, which illustrates the image that the plate lines redirect the force of vortex rebound into premature vortex decay, at least directly above the plates. Apart from the plates, the rebound is much more pronounced, pointing at the three-dimensional nature of the flow. In the A380 case, it is $p d=1.0$, where vortices descend less and rebound higher, corresponding to the weaker decay. Note that the propagating vortex helix (Figs. $\underline{4}$ and 5) reaches the domain boundaries at $t=60 \mathrm{~s}$ or $t=70 \mathrm{~s}$, depending on the set in time of the decay process. Thus, one has to be careful when interpreting results at later times.

\section{B. Aspect Ratio}

Figure 7 depicts the circulation evolution resulting from the aspect ratio variation. Particularly, the PRD initialization seems to be shifted to earlier times with a decreasing aspect ratio. Here, the vortex reorganization effect can be observed with different intensity for the A340 most pronounced for $a r=0.5,0.25$. In both cases, there is a tendency for a stronger reorganization effect with a smaller aspect ratio. Furthermore, the initial decay rate is larger with a smaller aspect ratio. These two effects seem to counteract and to level out after $50 \mathrm{~s}$ for the A340: first, above the obstacle, and about $10 \mathrm{~s}$ later in a distance of $b_{0}$. The initial time of the PRD is much more aspect-ratio
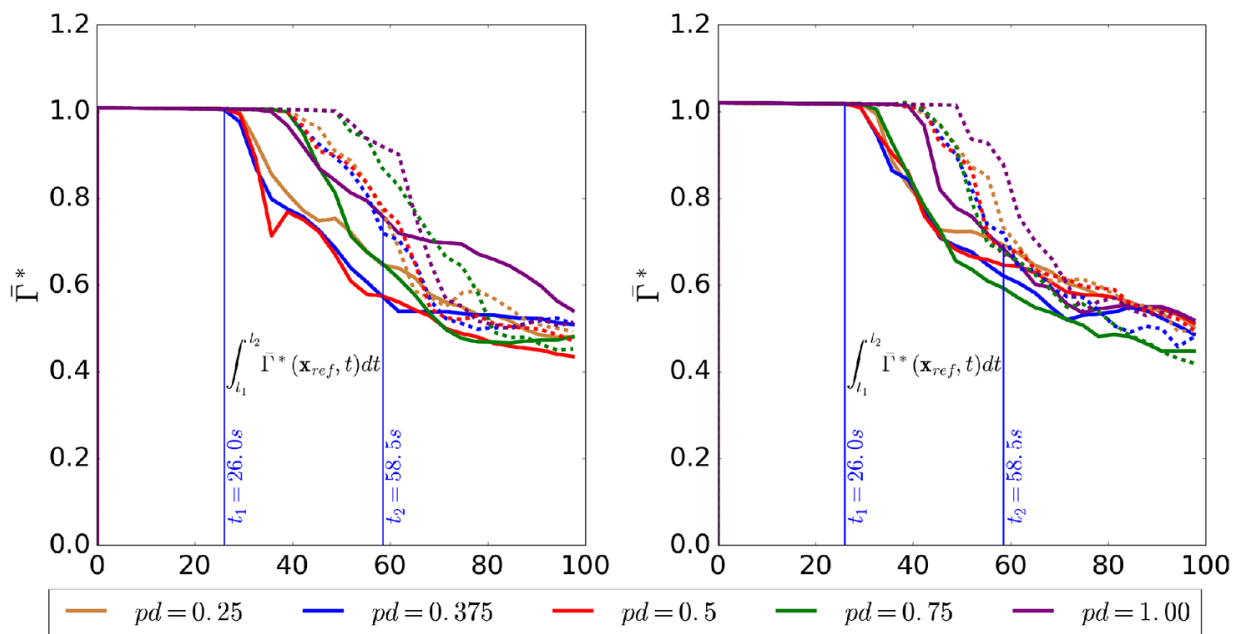

Fig. 3 Normalized circulation decay at $x=0$ (solid lines) and $x=b_{0}$ (dotted lines): A340 (left), and A380 (right). 


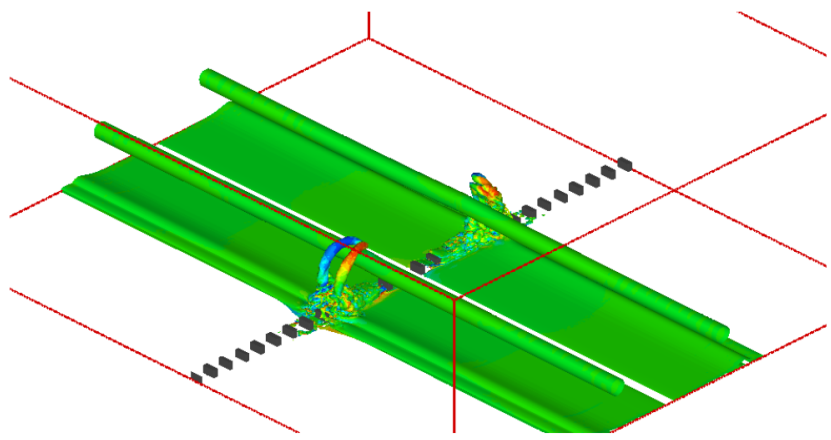

a) $p d=0.25 b_{r e f}, t=32.5 \mathrm{~s}$

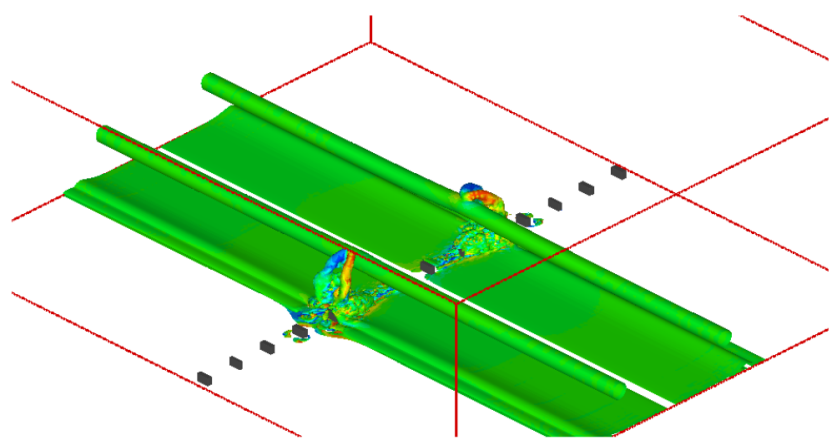

c) $p d=0.5 b_{r e f}, t=32.5 \mathrm{~s}$

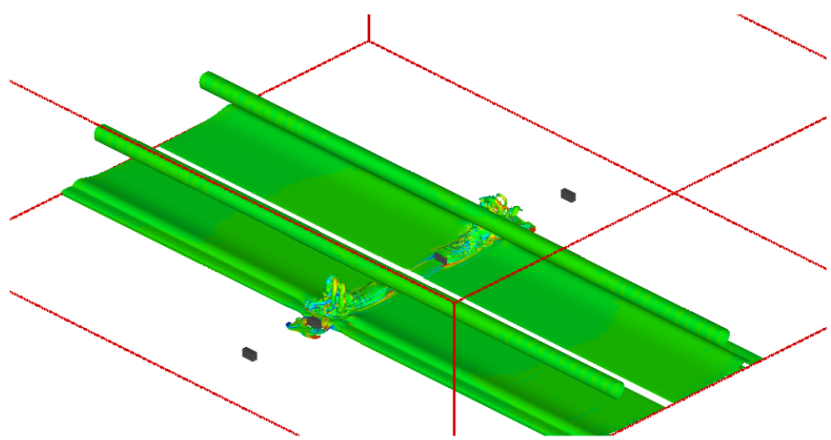

e) $p d=0.5 b_{\text {ref }}, t=32.5 \mathrm{~s}$

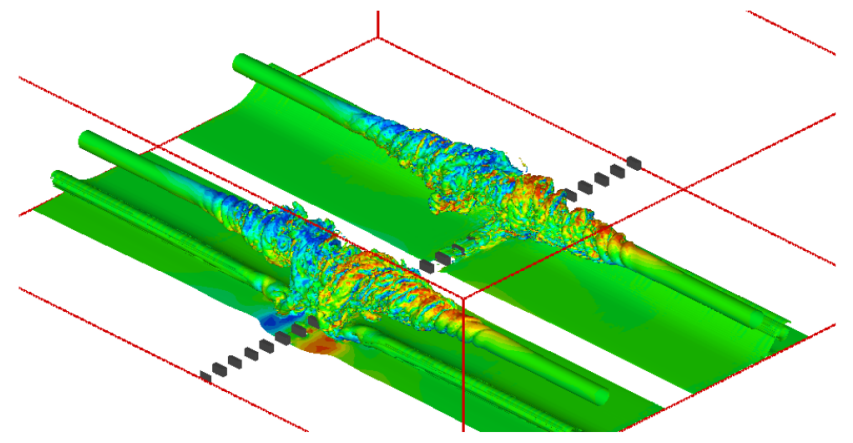

b) $p d=0.25 b_{r e f}, t=48.75 \mathrm{~s}$

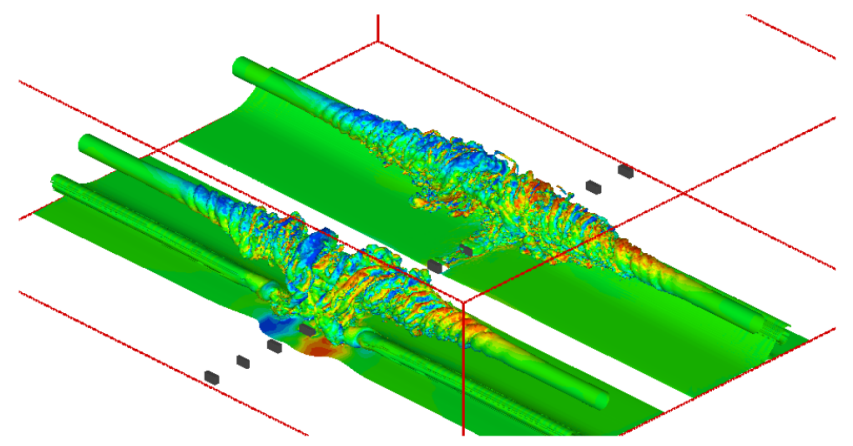

d) $p d=0.5 b_{r e f}, t=48.75 \mathrm{~s}$

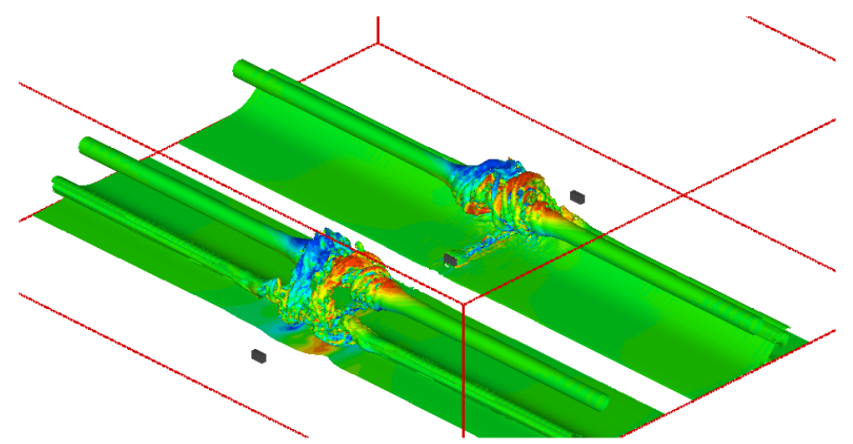

f) $p d=0.5 b_{r e f}, t=48.75 \mathrm{~s}$

Fig. 4 A340 wake-vortex evolution with plate line, three plate distances, and two vortex ages.

sensitive than it is for plate distance variation. Interestingly, both wake-vortex systems of the A340 and A380 show this behavior similarly. Note, that the aspect ratio affects the A380 more strongly, as the vortices are stronger, having the same ground distance, and therefore interact more vigorously.

Comparing two characteristic vortex ages of 32.5 and $48.75 \mathrm{~s}$ of the flowfield of our simulations, shown in Figs. 8 and 9, we observe that the generation of the SVS above the plates is strongly affected by the aspect ratio. A higher aspect ratio leads to close branches of the secondary vortex loop. The hairpin vortex rises more slowly than the reference case. The turbulent vortex interaction at $48.75 \mathrm{~s}$ is more pronounced for a distinct omega loop generated by a small aspect ratio. Seemingly, the secondary vortex strength is influenced by the aspect ratio. This can be explained by a weakening of the two branches of the vortex loop by mutual interaction. A small aspect ratio implies close branches, and consequently mutual interaction. Hence, the initial strong decay rate, observable particularly for the A340, is a direct consequence of the maturity of the secondary vortex loop. There seems to be a clear tendency of faster decay with the smaller aspect ratio. However, the quantification of that tendency is not obvious, and it requires a suitable decay measure. Again, trajectories are plotted; see Fig. 10. We observe a correlation of weaker vortex rebound in the case of a stronger vortex decay.

Summarizing, we observe that the plate distance as well as the aspect ratio influence the decay rates for both aircraft types. The aspect ratio effects are much more pronounced in the A380 case. Smaller aspect ratios seem to be more effective. Yet, from Figs. 3 and 7, we can hardly derive an optimum, even in the case of one fixed parameter, let alone for the parameter combination of $p d$ and $a r$.

\section{Objective Function}

As observed in the previous section, the circulation above the plate line at a fixed vortex age is a rather inaccurate measure for design optimization. As a more suitable measure for vortex decay than circulation at a certain time step, we define the objective function as

$$
Z(\boldsymbol{x})=\frac{1}{t_{2}-t_{1}} \int_{t_{1}}^{t_{2}} \Gamma^{*}(\boldsymbol{x}, t) \mathrm{d} t
$$

Here, $t_{1}=26.0 \mathrm{~s}$ denotes the moment when the plate-line effect starts in the reference case and $t_{2}=58.5 \mathrm{~s}$, which is the time step when the helical secondary vortices leave the computational domain, resulting in overrun effects due to the periodic boundaries (see, i.e., Fig. 3), which is actually close to the minimal radar separation between subsequent aircraft. The integrated circulation overcomes the shortcomings of the fluctuating circulation at instant time steps. With normalization by the respective time span, one can interpret the objective function as a mean circulation during the phase of rapid decay. The parameter domain consists of 


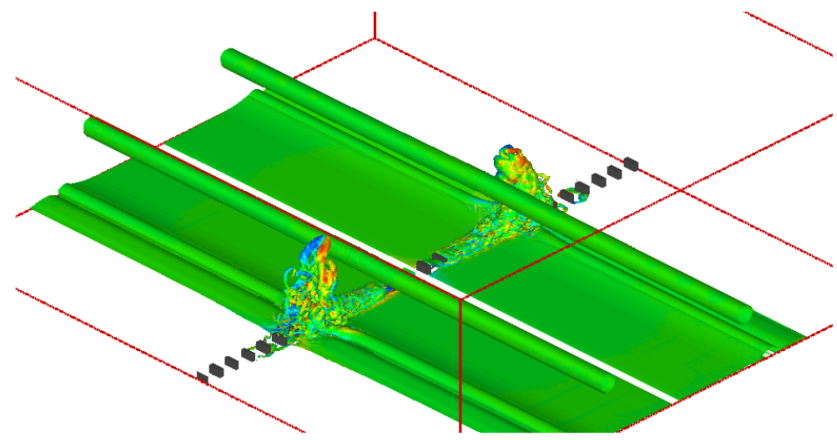

a) $p d=0.25 b_{r e f}, t=32.5 \mathrm{~s}$

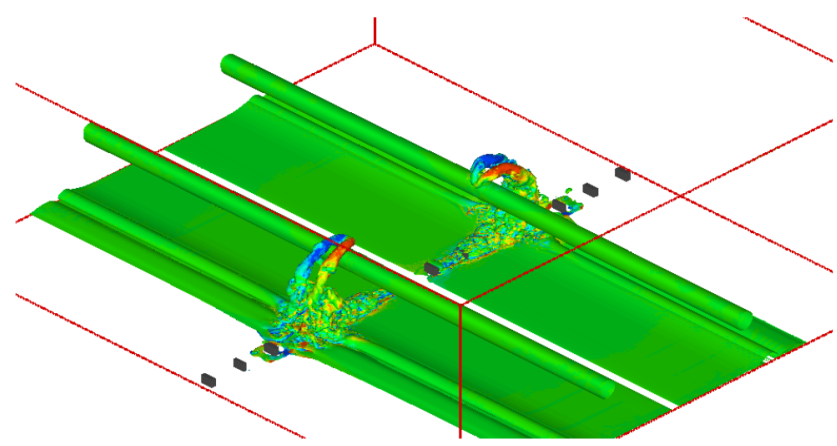

c) $p d=0.5 b_{r e f}, t=32.5 \mathrm{~s}$

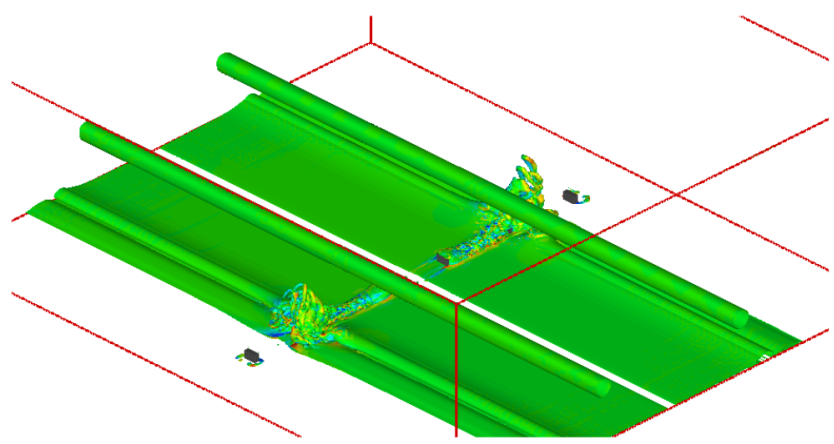

e) $p d=1.0 b_{r e f}, t=32.5 \mathrm{~s}$

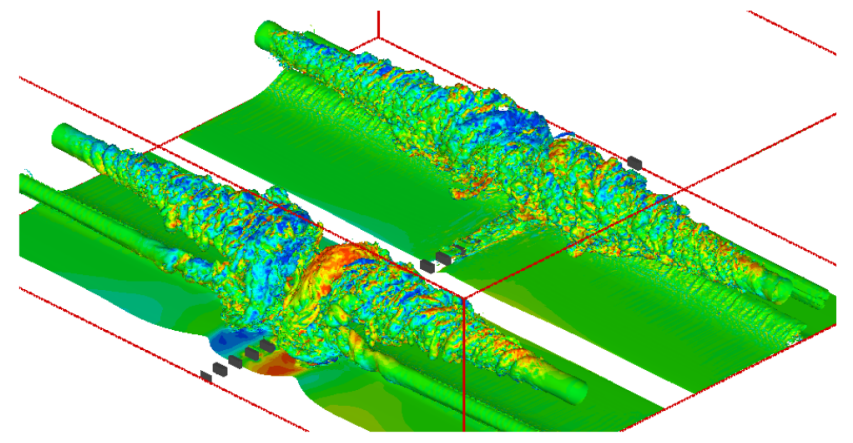

b) $p d=0.25 b_{r e f}, t=48.75 \mathrm{~s}$

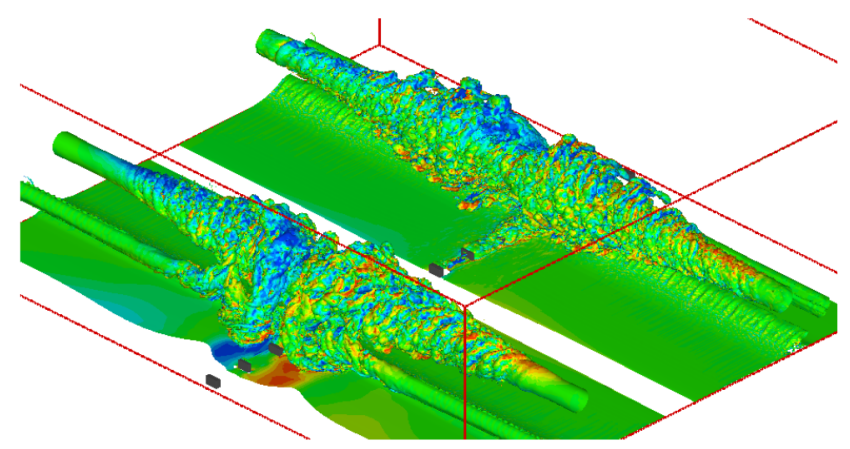

d) $p d=0.5 b_{r e f}, t=48.75 \mathrm{~s}$

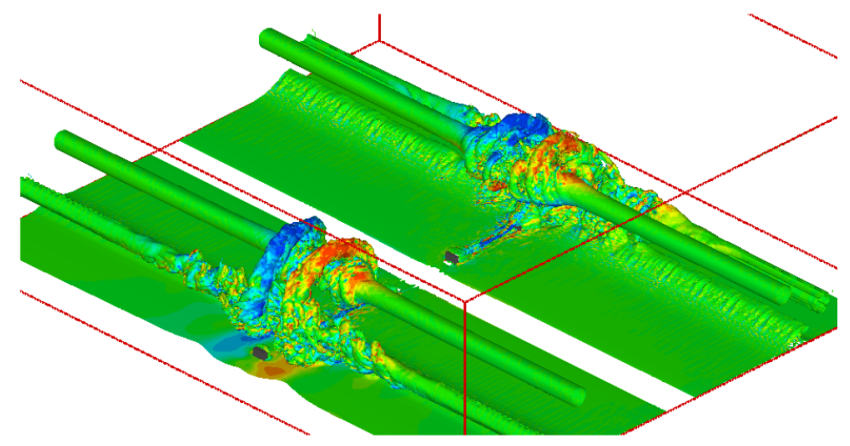

f) $p d=1.0 b_{r e f}, t=48.75 \mathrm{~s}$

Fig. 5 A380 wake-vortex evolution with plate line, three plate distances, and two vortex ages.
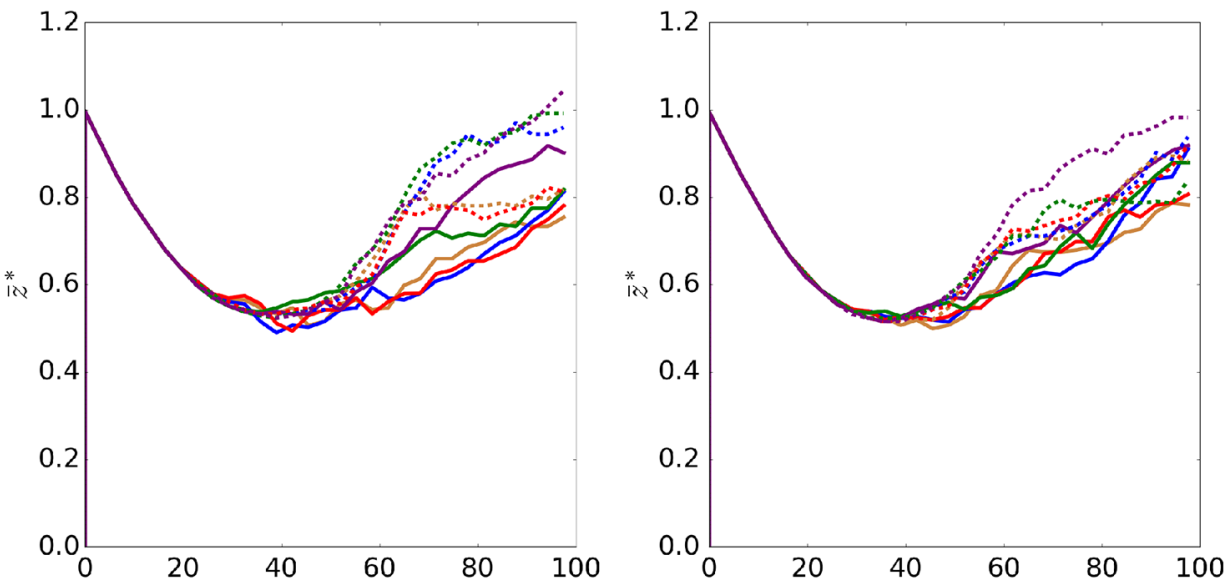

$-p d=0.25-p d=0.375-p d=0.5-p d=0.75-p d=1.00$

Fig. 6 Normalized vortex height at $x=0$ (solid lines) and $x=b_{0}$ (dotted lines): A340 (left), and A380 (right). 


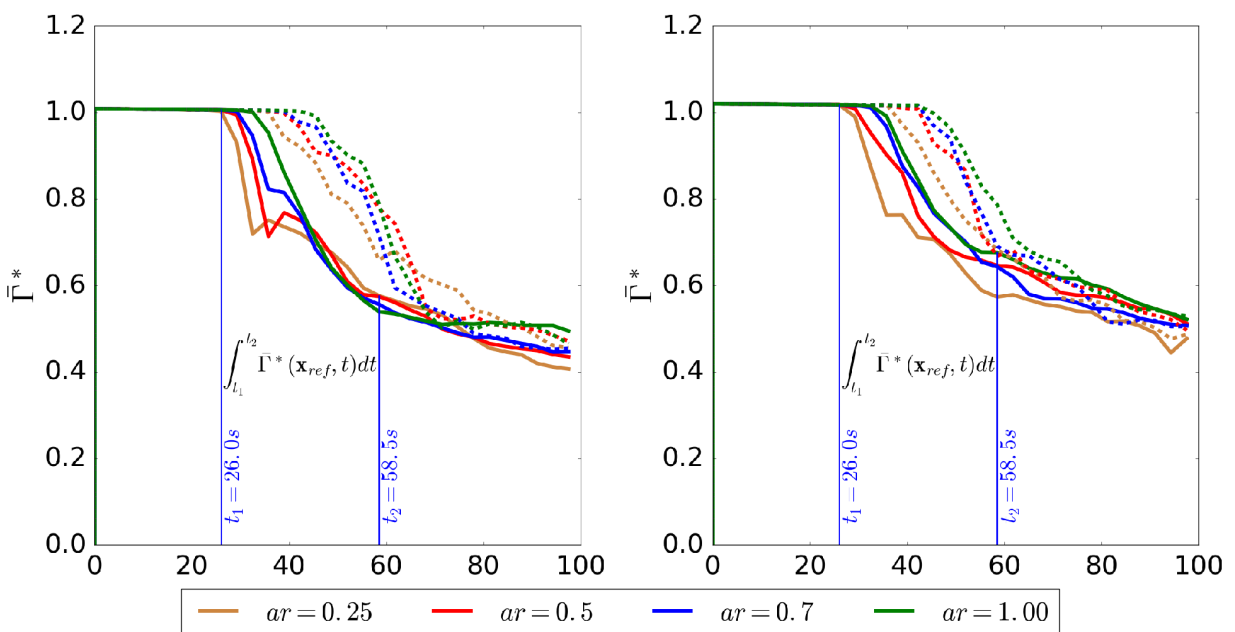

Fig. 7 Normalized circulation decay at $x=0$ (solid lines) and $x=b_{0}$ (dotted lines): A340 (left), and A380 (right).

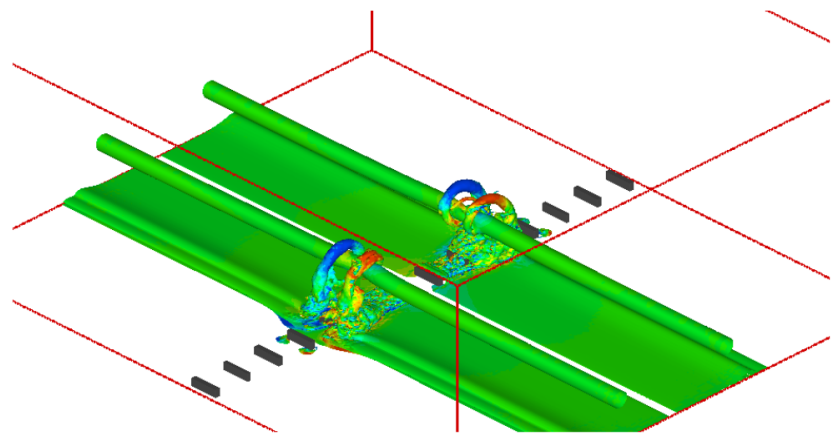

a) $\operatorname{ar}=0.25, t=32.5 \mathrm{~s}$

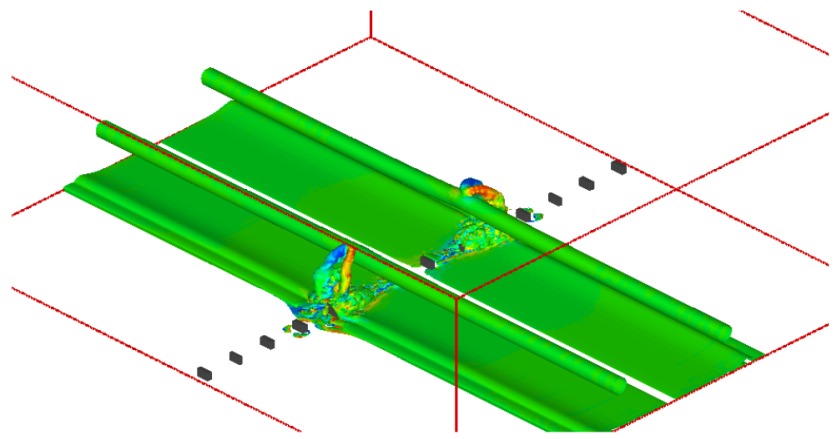

c) $a r=0.5, t=32.5 \mathrm{~s}$

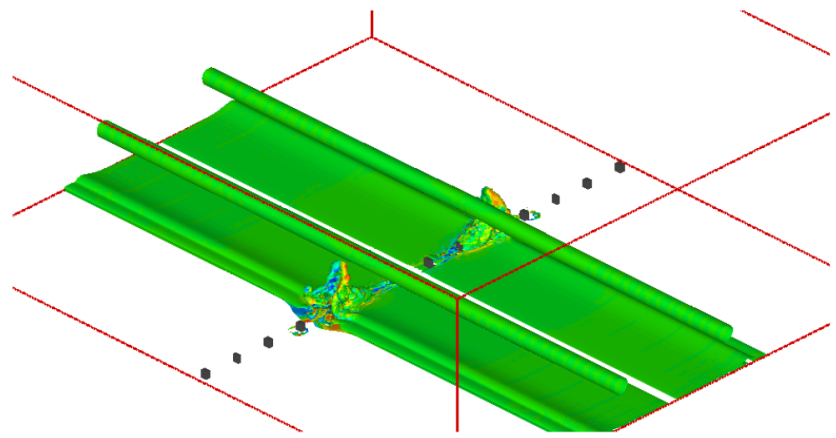

e) $a r=0.5, t=32.5 \mathrm{~s}$

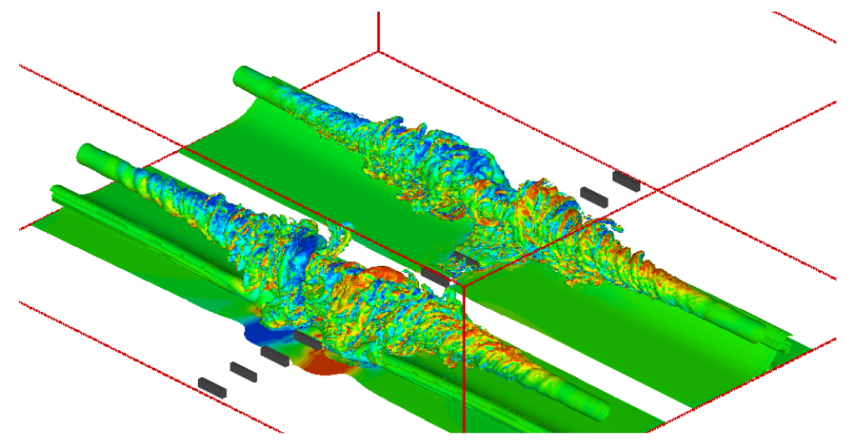

b) $a r=0.25, t=48.75 \mathrm{~s}$

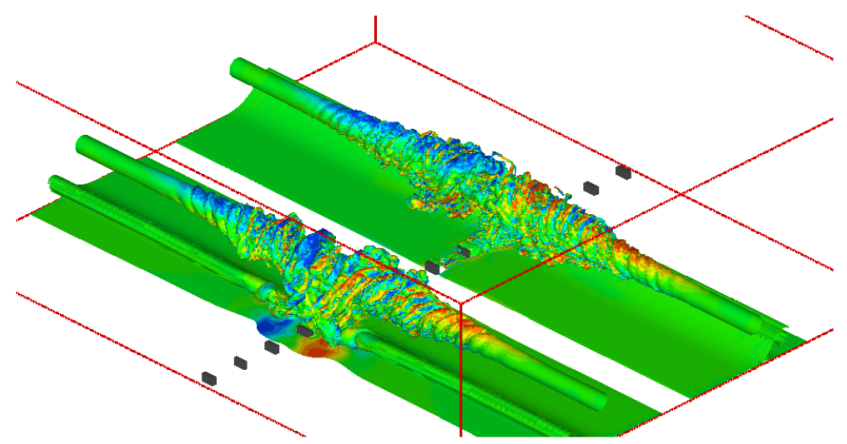

d) $a r=0.5, t=48.75 \mathrm{~s}$

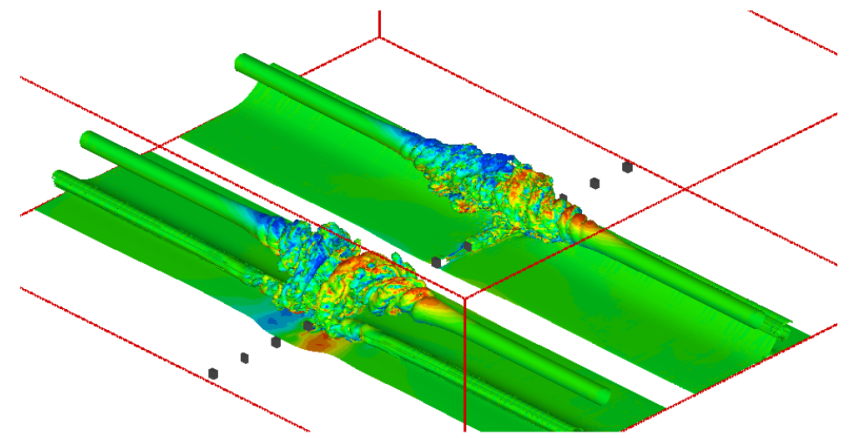

f) $a r=1.0, t=48.75 \mathrm{~s}$

Fig. 8 A340 wake-vortex evolution with plate line, three aspect ratios, and two vortex ages. 


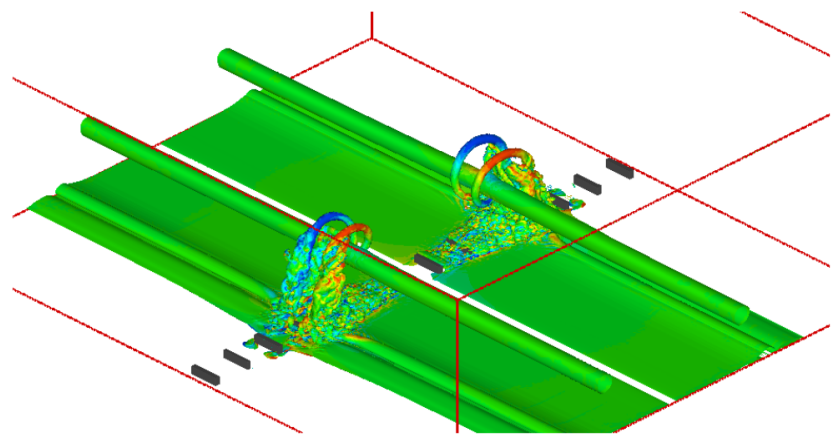

a) $a r=0.25, t=32.5 \mathrm{~s}$

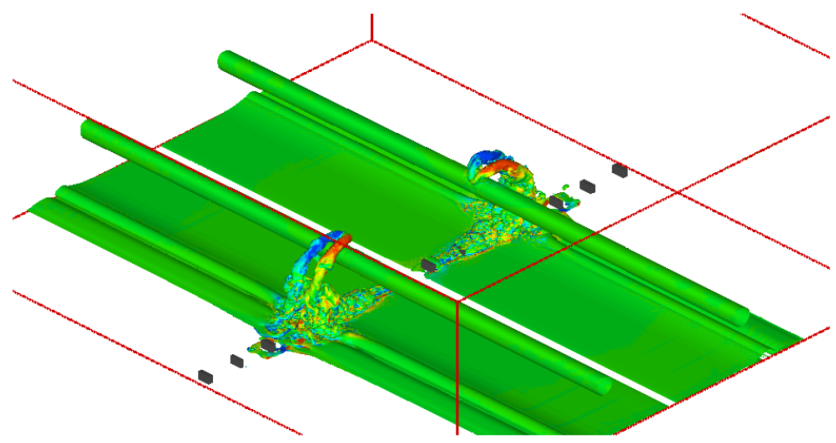

c) $\operatorname{ar}=0.5, t=32.5 \mathrm{~s}$

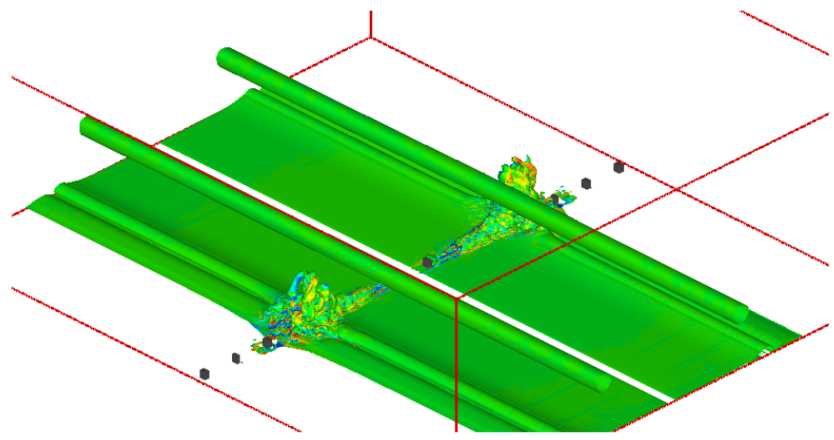

e) $a r=1.0, t=32.5 \mathrm{~s}$

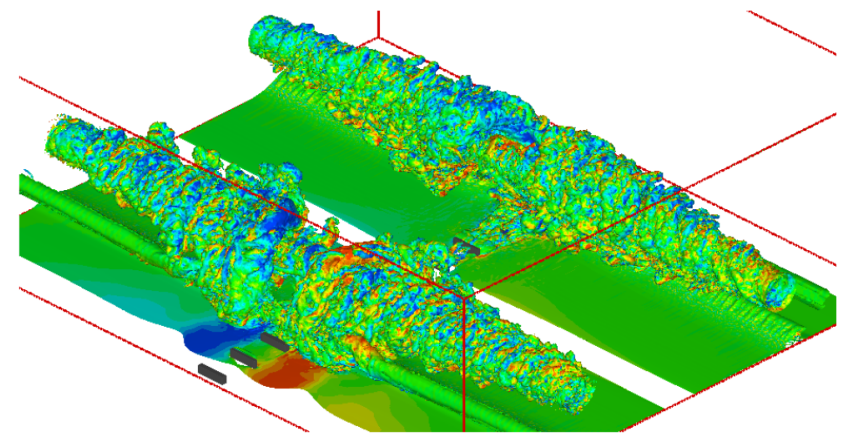

b) $a r=0.25, t=48.75 \mathrm{~s}$

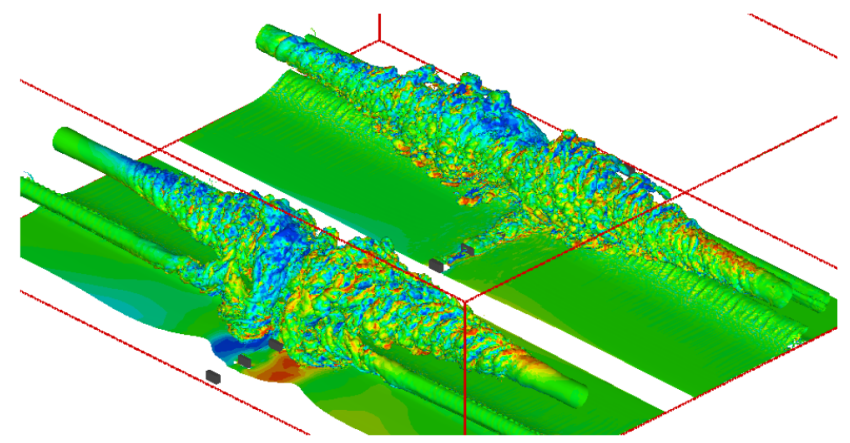

d) $a r=0.5, t=48.75 \mathrm{~s}$

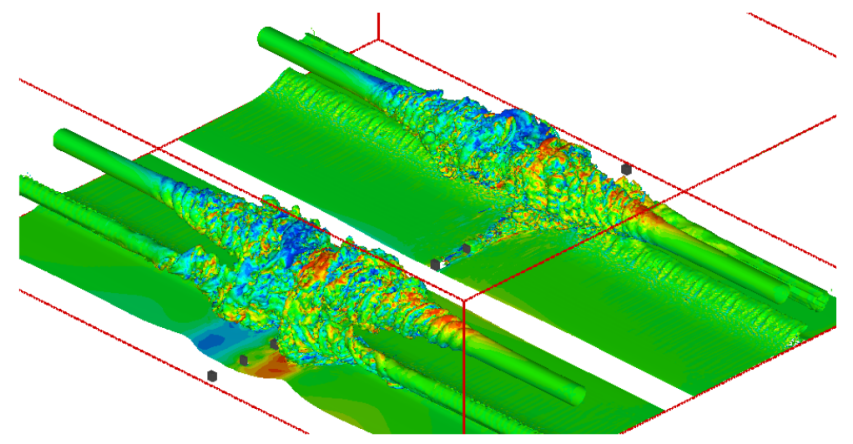

f) $a r=0.5, t=48.75 \mathrm{~s}$

Fig. 9 A380 wake-vortex evolution with plate line, three aspect ratios, and two vortex ages.

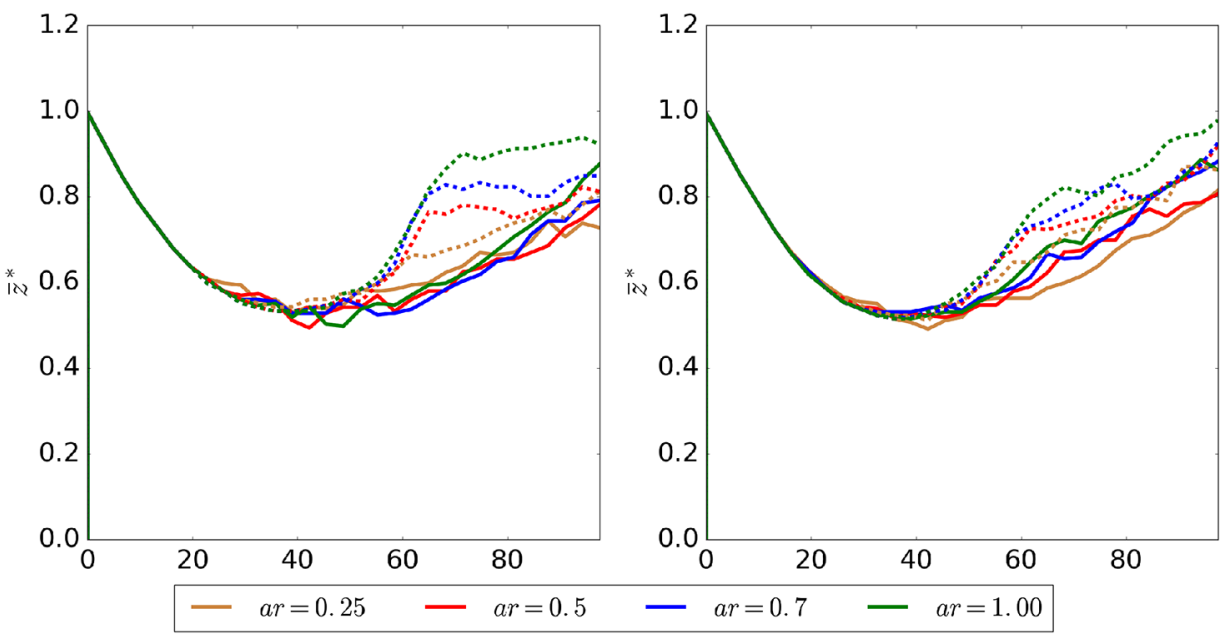

Fig. 10 Normalized vortex height at $x=0$ (solid lines) and $x=b_{0}$ (dotted lines): A340 (left), and A380 (right). 
Table 4 Examined values of objective function from simulations (A340)

\begin{tabular}{lccccccccc}
\hline \hline Case & 1 & 3 & 5 & 7 & 9 & 11 & 13 & 15 & 17 \\
\hline ar & 0.500 & 0.500 & 0.500 & 0.500 & 0.500 & 0.250 & 0.700 & 1.000 & 0.700 \\
$p d$ & 0.500 & 0.250 & 0.375 & 0.750 & 1.000 & 0.500 & 0.500 & 0.500 & 0.750 \\
$Z_{i}$ & 0.687 & 0.765 & 0.713 & 0.858 & 0.887 & 0.684 & 0.702 & 0.735 & 0.851 \\
\hline \hline
\end{tabular}

Table 5 Examined values of objective function from simulations (A380)

\begin{tabular}{lccccccccc}
\hline \hline Case & 2 & 4 & 6 & 8 & 10 & 12 & 14 & 16 & 18 \\
\hline ar & 0.500 & 0.500 & 0.500 & 0.500 & 0.500 & 0.250 & 0.700 & 1.000 & 0.700 \\
$p d$ & 0.500 & 0.250 & 0.375 & 0.750 & 1.000 & 0.500 & 0.500 & 0.500 & 0.750 \\
$Z_{i}$ & 0.753 & 0.777 & 0.747 & 0.747 & 0.867 & 0.690 & 0.795 & 0.810 & 0.755 \\
\hline \hline
\end{tabular}

the plate-line parameter aspect ratio and plate distance $[\boldsymbol{x}=(a r, p d)] . \Gamma^{*}(\boldsymbol{x}, t)$ is derived from the presented simulations with different plate-line parameters $\boldsymbol{x}_{i}, i=1, \ldots, n$ and presented individually for the two investigated aircraft types. For optimization purposes, we use a kriging model for building a continuous two-dimensional surface. The simulation results for the objective function $Z$ of the plate-line effect for the A340 as well as the A380 are listed in Table $\underline{4}$ and $\underline{5}$.

\section{Kriging Model}

We analyze the semivariograms for three to six recommended lags [38]. The resulting points $\left\{h_{i}, \gamma\left(h_{i}\right)\right\}$ ae fitted by a Gaussian model using a least-squares approximation to determine each model's optimal parameters. The Gaussian model is widely used $[39,40]$ and defines the variogram and covariance function according to $\overline{\mathrm{Eqs}}$. (ㅁ) and (9).

We examined the three different quality measures $Q_{1}, Q_{2}$ and $c R$ given in Eqs. (10), (14), and (16). The results are listed in Table $\underline{6}$. Different models were also tested. The spherical as well as a linear model yielded worse results in terms of the quality measures $Q_{1}, Q_{2}$, and $c R$. The Gaussian model turned out to be the most accurate, as it was particularly effective for stationary random functions $Z(\boldsymbol{x})$. Different numbers of lags led to similar results for interpolation with similar quality measures. Therefore, the chosen variogram model was stable. For convenience, we chose five lags for the Gaussian model. Also, for convenience, the same number of lags was taken for the A340 as well as the A380 case, since both datasets described a similar process.
Table 6 Gaussian model for semivariogram, parameters, quality measures

\begin{tabular}{lcccccc}
\hline \hline Aircraft & Lags & $S$ & $K$ & $Q_{1}$ & $Q_{2}$ & $c R$ \\
\hline A340 & 3 & $7.0 \mathrm{E}-03$ & 0.35 & 0.30 & 0.93 & $2.51 \mathrm{E}-3$ \\
& 4 & $6.4 \mathrm{E}-03$ & 0.30 & 0.34 & 0.72 & $2.50 \mathrm{E}-3$ \\
& 5 & $6.6 \mathrm{E}-03$ & 0.34 & 0.32 & 0.87 & $2.45 \mathrm{E}-3$ \\
& 6 & $6.5 \mathrm{E}-03$ & 0.29 & 0.34 & 0.69 & $2.55 \mathrm{E}-3$ \\
A380 & 3 & $3.1 \mathrm{E}-03$ & 0.37 & 0.50 & 2.77 & $2.94 \mathrm{E}-3$ \\
& 4 & $3.2 \mathrm{E}-03$ & 0.4 & 0.50 & 4.15 & $3.57 \mathrm{E}-3$ \\
& 5 & $3.1 \mathrm{E}-03$ & 0.4 & 0.51 & 4.39 & $3.57 \mathrm{E}-3$ \\
& 6 & $3.4 \mathrm{E}-03$ & 0.42 & 0.50 & 4.81 & $3.90 \mathrm{E}-3$ \\
\hline \hline
\end{tabular}

The modeling of the variogram data is depicted in Fig. 11. Here, the raw variogram data $\gamma(h)$ are shown as points together with the experimental variogram $\hat{\gamma}\left(h_{k}\right)$, as well as the Gaussian model. In the A340 case, the semivariogram is steeper than in the A380 case. The raw variogram data are much more concentrated close to the model. This results in a smaller standard deviation for the interpolation. Following Eqs. (13) and (15), with a total number of $n=36$ combination pairs in the $\mathrm{A} \overline{340}$ as well as the $\mathrm{A} 380$ case, we have

$$
\frac{2}{\sqrt{35}}=0.33, \quad 1-\frac{2 \sqrt{2}}{\sqrt{35}}=0.52, \text { and } 1+\frac{2 \sqrt{2}}{\sqrt{35}}=1.48
$$

Hence, we can state that, in the A340 case, the kriging model is of good quality with respect to the quality measures $Q_{1}$ and $Q_{2}$. In the A380 case, the quality measures yield an unsatisfactory result. This is due to an underestimation of the error $\sigma_{k}$; see Eq. (11). However, we stick to that model, as it shows good results for interpolation, as well as in the A380 case, keeping in mind that the error $\sigma$ is underdetermined by the Gaussian model.

\section{E. Kriging Interpolation of the Parameter Space}

After determining the model parameters, the kriging interpolation of the two-dimensional parameter space is performed. Onedimensional kriging interpolation, choosing one parameter as constant at 0.5 , shows the quality of the kriging prediction. The interpolated objective function as a stochastic process is plotted in Fig. 12. The mean value as well as the $95 \%$ interval using $\hat{\Gamma}^{*}-2 \sigma$ of the Gaussian process are plotted. In both cases, the kriging interpolation is satisfactory inside the simulated parameter range. The extrapolation seems to overestimate expected values of the objective function. Especially for small aspect ratios, there is no evidence that the objective function increases. This is important for the interpretation of the results, in view of the operational
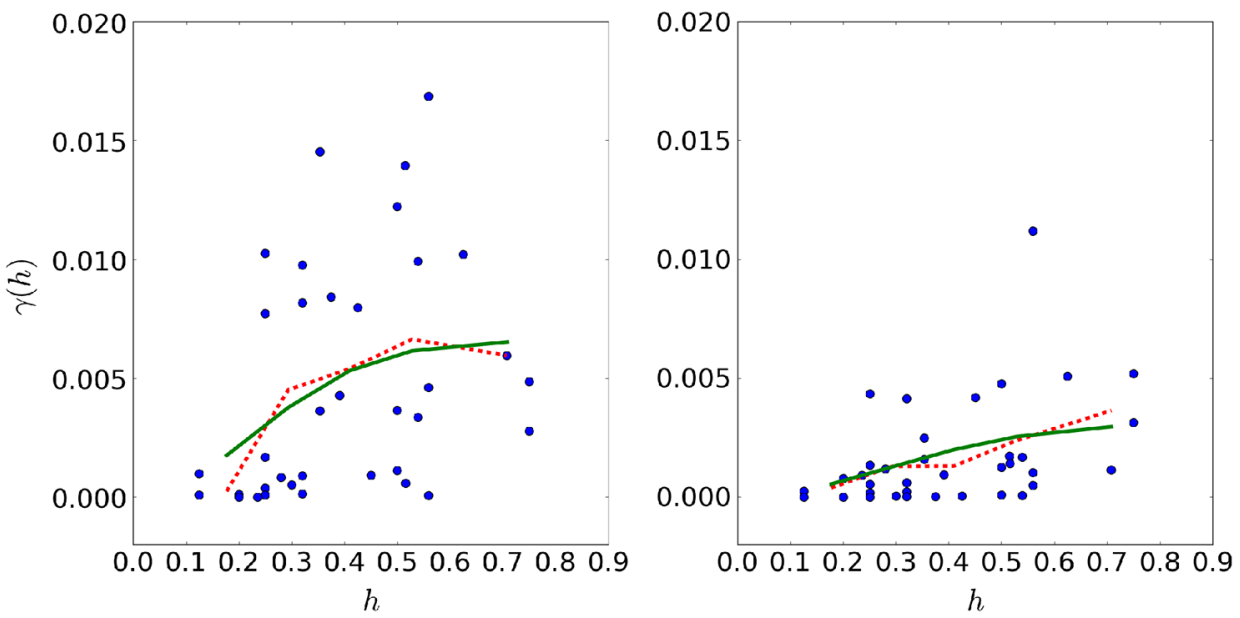

Fig. 11 Raw variogram (dots), experimental variogram (dotted lines), and model variogram (solid lines): A340 (left), and A380 (right). 

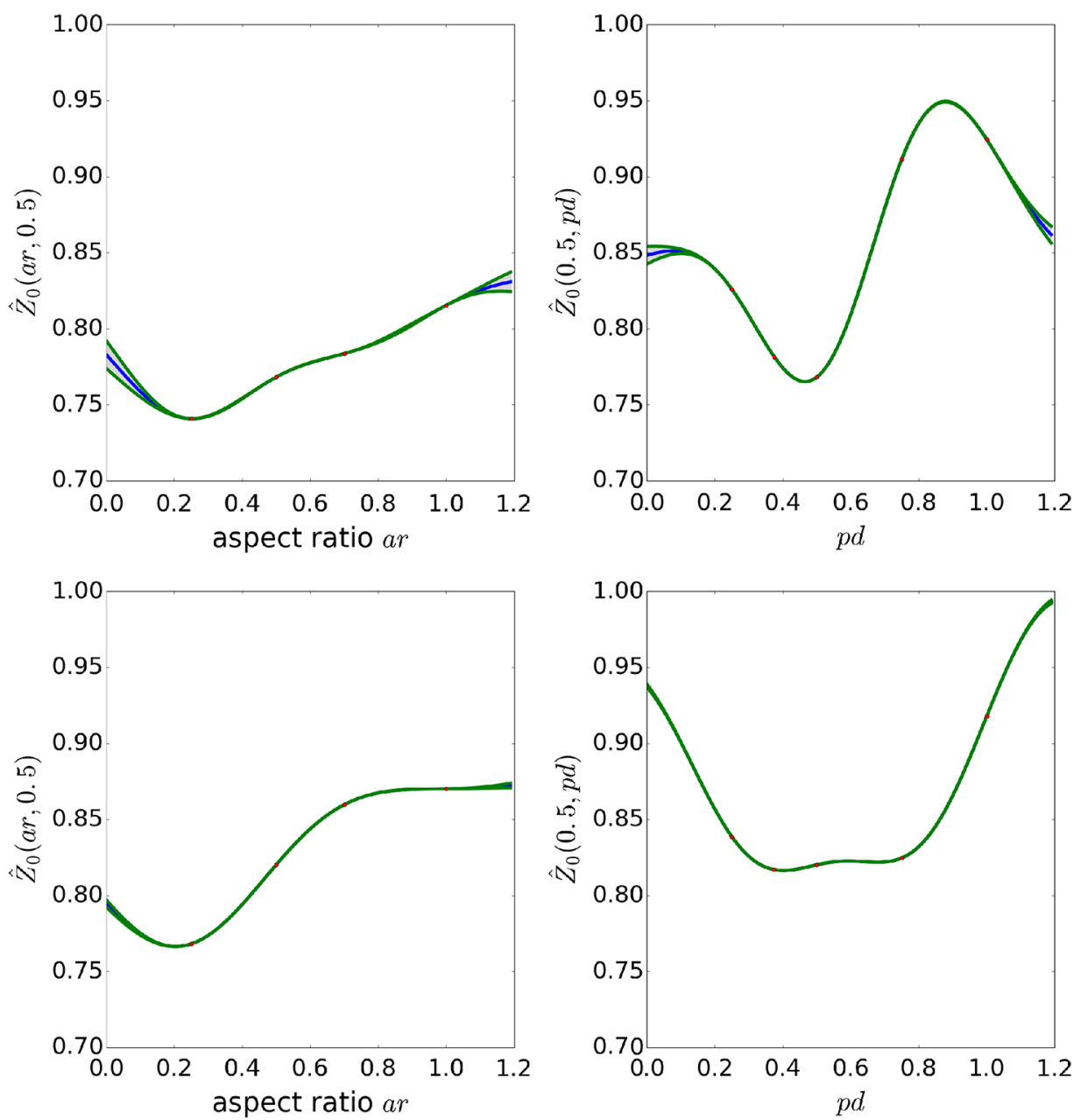

Fig. 12 One-dimensional kriging interpolation along $a r$ axis (left) and $p d$ axis (right): A340 (top), and A380 (bottom).

practicability. Note that no arbitrary small aspect ratios can be realized at an airport. For a fixed plate height, this will imply impracticably wide plates.

The two-dimensional interpolation of the entire parameter space (see Fig. 13) reveals the complexity of the objective function as well as the challenge to optimize the plate-line design. The mean value of the Gaussian process is plotted to the left, and the standard deviation is to the right. This yields estimations of the objective function together with uncertainty measures of the predicted region. The area enclosed by the simulation points gives much more accurate results. Close to the corners and edges, the estimations are becoming inaccurate. It seems that the kriging interpolation gives better results for the A380 case, as the standard deviation is lower. However, as the quality measure of the used model is rather poor due to underestimated $\sigma$, one should interpret that plot qualitatively. In both cases, a minimum region can be located in the area of $(a r, p d)=(0.25,0.5)$. That the optimal regions partly coincide is an important result for the operational use of plates. Note further that, in the A380 case, the optimal region clearly deviates from the simulated points still lying in the well-predicted region with a small standard deviation. The actual minima are listed in Table 7.

The consideration of the lower and upper 95\%-confidence interval gives further insight in the uncertainties of the interpolated field; see Fig. 14e. The upper 95\%-confidence interval is an upper bound for the plate-line effect. With a $95 \%$ probability, a plate configuration with $(a r, p d) \in[0.2,0.7] \times[0.4,0.5]$ leads to an averaged vortex decay during the PRD to 0.79 in the $\mathrm{A} 340$ case. The same estimate in the A380 case can be done for the region $(a r, p d) \in[0.15,0.35] \times[0.35,0.55]$. The lower 95\%-confidence interval indicates whether other (local) minima exist; see Fig. 14. Other (local) minima can be excluded this way, i.e., in the A380 case in the critical region around $(a r, p d)=(0.8,0.8)$.

The operational construction of plate lines further depends on various parameters and constraints. First, the localizer of the instrument landing system should not be disturbed by the plates. Second, the runway lighting should be visible, and it should not be hidden by the plates. Ideally, every single light should be visible from any possible aircraft position. Third, obstacle clearance and frangibility criteria must be met; that is, the material and position should not decrease safety in the case of runway excursions. Another aspect for the design at a particular airport may be the aircraft types that cause most encounters.

This work provides an important methodology for designing the operational layout of plate lines. Even if the optimal region in the parameter space cannot be achieved by some constraints listed previously, one can estimate the plate-line effect in adjacent regions to the optimum parameter combination. We observe that the surface and the slopes of the objective function are nontrivial. Therefore, this plot provides the most effective parameter to be adjusted in the operational setting.

$\begin{gathered}\text { Table 7 } \\
\text { found by kriging } \\
\text { interpolation }\end{gathered}$
\begin{tabular}{cccc}
\multicolumn{3}{c}{ Minima } \\
\hline \hline$a / c$ & $a r$ & $p d$ & $\hat{Z}$ \\
\hline $\mathrm{A} 340$ & 0.27 & 0.49 & 0.728 \\
$\mathrm{~A} 380$ & 0.20 & 0.43 & 0.742 \\
\hline \hline
\end{tabular}




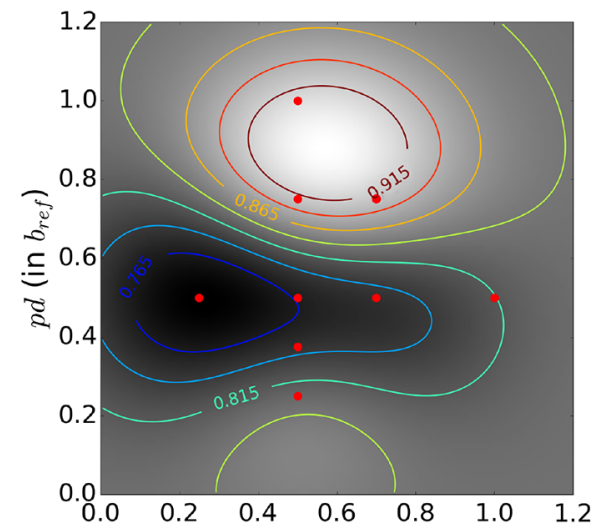

a)

ar (height/width)

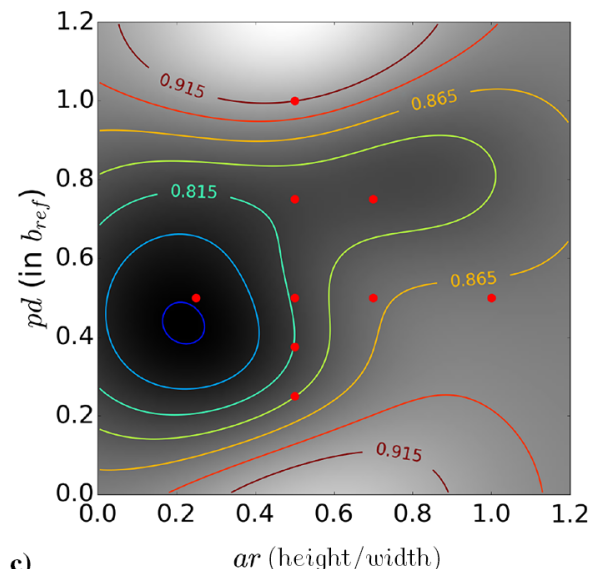

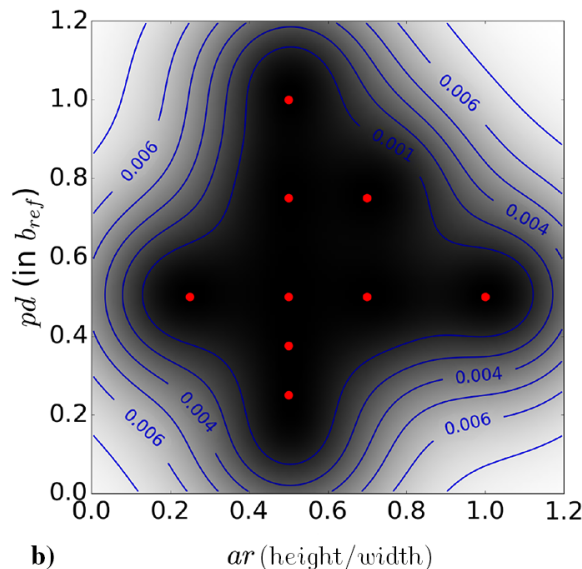

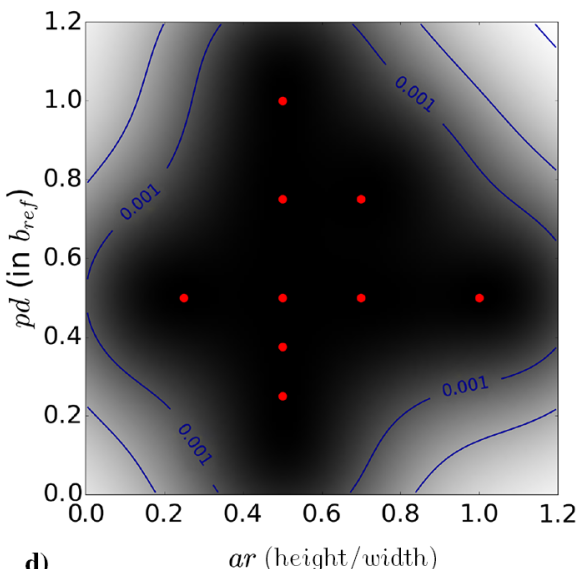

Fig. 13 Objective function (kriging estimator) and standard deviation: A340 (top), and A380 (bottom).
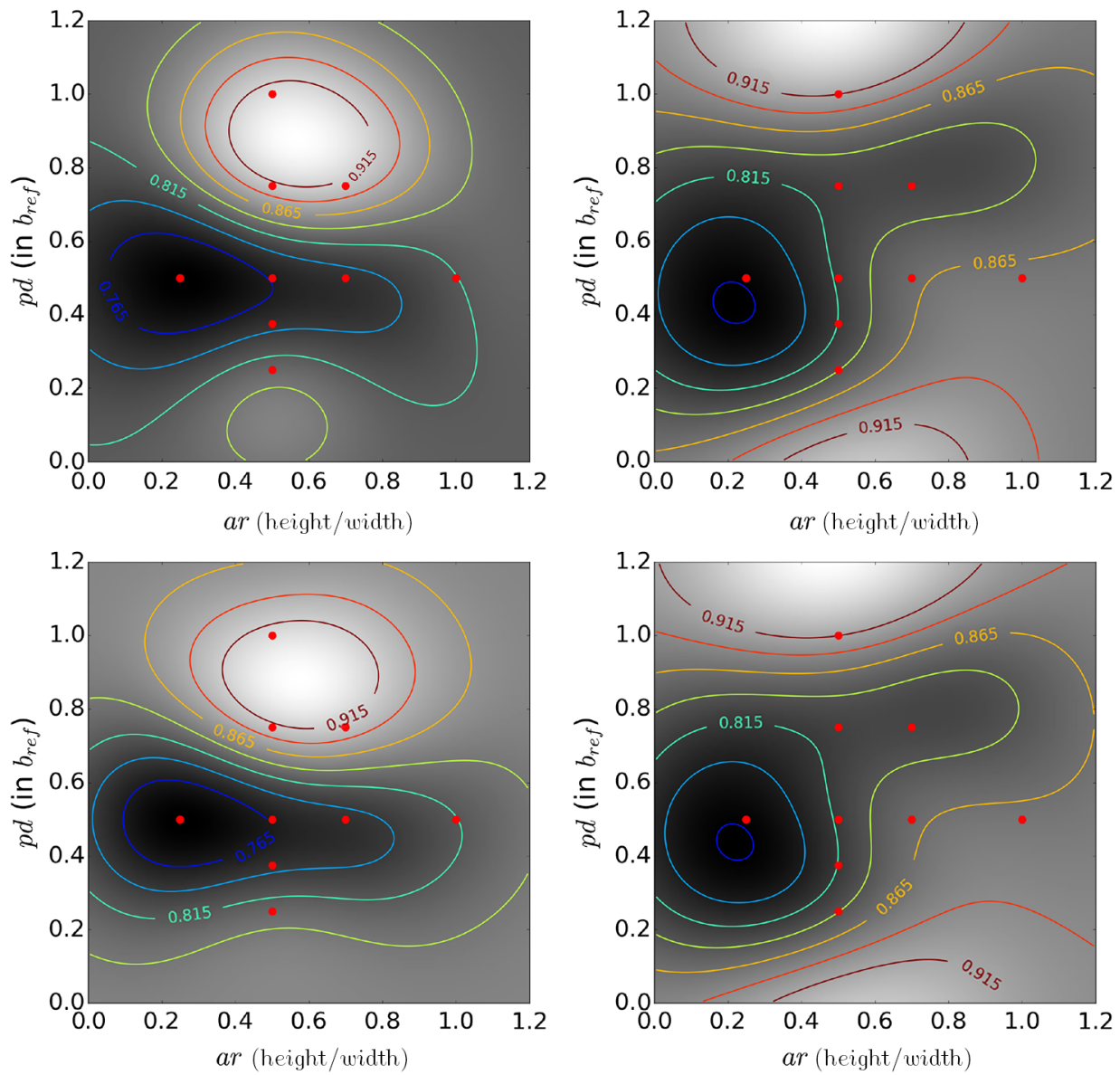

Fig. 14 Lower (top) and upper (bottom) 95\%-confidence intervals for kriging models: A340 (left), and A380 (right). 


\section{Conclusions}

To optimize the operational design of plate lines, a study was performed based on two independent methods. First, large-eddy simulation was used to simulate the wake-vortex evolution in ground proximity for different parameter configurations. Second, the entire parameter space was statistically interpolated using kriging, which is a Gaussian regression algorithm. The aspect ratio and plate distance, as the most important design parameters, were analyzed for the wake vortices of two different aircraft types: the A340, as well as the A380.

In the LES, fully rolled-up wake vortices are initialized using a Lamb-Oseen vortex model. The flowfield is analyzed with respect to vortex trajectories as well as circulation.

It is found that the decay process is in fact moderately dependent on both parameters, at least within a region, where the optimum is expected. Instantaneous circulation does not provide a direct answer to the question of which parameter setting is most effective, as the circulation curves feature substantial scatter. Therefore, an appropriate temporal average of the circulation is applied as the objective function.

With the stochastical interpolation method, so-called kriging, probabilistic envelopes, and estimates are given for the entire parameter space, which is spanned by the LES. From theoretical considerations, the problem of the existence of a global minimum is well posed concerning one parameter, the plate distance) as for extremal values (i.e., $p d \rightarrow \infty$ as well as $p d \rightarrow 0$ ), the objective function is not optimal. The plate-line performance with respect to the aspect ratio is not clear ors. As it turns out from the chosen parameter set, $a r \rightarrow 0$ optimizes the plate-line effect, at least with respect to the applied objective function. However, the recovery effects of the circulation appear particularly in that region. That mechanism is not fully understood. The operational feasibility constrains the parameter space in this direction. Therefore, optimal regions for both aircraft types can still be provided.

As a result, from the kriging method, it was found that, in the A340 case, the optimal parameter combination was not localized; whereas in the A380 case, a statistical relevant optimum could be found. Moreover, it could be deduced that the optimal parameters for the A380 were also well suited for smaller aircraft like an A340. Statistical estimates were provided for the probability of the results, in the sense that confidence intervals were determined. In addition, this yielded the best estimates for the effectiveness of the plate-line parameters, deviating from the simulated discrete parameter combinations.

In summary, the introduction of obstacles at the ground generates secondary vortex structures that approach the primary vortices actively and rapidly spreading disturbances along the primary vortices, leading to accelerated vortex decay in ground proximity. The plate-line design mostly depends on two parameters: the plate distance and plate aspect ratio. The optimal parameters depend on the aircraft size. This study has shown that, for the A380, a clear minimum is in the region of a plate aspect ratio of $a r=0.25$ $(4.7 \times 19 \mathrm{~m})$, which is also suitable for smaller aircraft like the A340. However, a plate length of about $10 \mathrm{~m}$ is more easy to establish at an airport and delivers reasonable performance.

\section{Appendix: Kriging}

Stochastic processes are used as a qualified estimator for the objective function. The use of a stochastic process in global optimization is called Bayesian global optimization. We briefly introduce the basics [44]. Kriging denotes a group of statistical techniques to interpolate the value $Z(x)$ of a random field $Z$ at an unobserved location $x$ based on observations $z_{i}=Z\left(x_{i}\right)$, $i=1, \ldots, n$ of the random field at nearby locations $x_{1}, \ldots, x_{n}$. Kriging computes the best linear unbiased estimator, $\hat{Z}(x)$ of $Z(x)$, based on a stochastic model of the spatial dependence quantified by expectation $\mu(x)=E[Z(x)]$ and the covariance function $c(x, y)$ of the random field.
The kriging estimator is given by a linear combination

$$
\hat{Z}(x)-\mu(x)=\sum_{i=1}^{n} w_{i}(x)\left[Z\left(x_{i}\right)-\mu\left(x_{i}\right)\right]
$$

$Z(x)$ is treated as a random field with a trend component $\mu(x)$ and a residual component $R(x)=Z(x)-\mu(x)$. Kriging estimates the residual at $x$ as the weighted sum of residuals at surrounding data points. The random field $R(x)$ has zero mean: $E[R(x)]=0$. The kriging weights $w_{i}$ are derived from the covariance function or the semivariogram. The goal is to determine the weights that minimize the variance of the estimator

$$
\begin{aligned}
\sigma^{2}(x) & :=\operatorname{Var}(\hat{Z}(x)-Z(x)) \\
& =\sum_{i, j=1}^{n} w_{i}(x) w_{j}(x) c\left(x_{i}, x_{j}\right)+\operatorname{Var}(Z(x))-2 \sum_{i=1}^{n} w_{i}(x) c\left(x_{i}, x\right)
\end{aligned}
$$

under the unbiasedness constraint $E[\hat{Z}(x)-Z(x)]=0$. We now assume that the covariance of $Z$ is stationary; it is a function of the lag $h$ but not of the position $x$.

We introduce the covariogram as well as the variogram

$$
\begin{aligned}
C_{R}(h) & =\operatorname{Cov}\{R(x), R(x+h)\}=E[R(x) \cdot R(x+h)], \\
\gamma(h) & =C_{R}(0)-C_{R}(h)
\end{aligned}
$$

which are generally derived from the input semivariogram model. There are several kriging variants; we will apply ordinary kriging in this study. The main assumption for ordinary kriging is that the mean is constant in a local neighborhood of each estimation point. That means $\mu\left(x_{i}\right)=\mu(x)$ for data that we are using to estimate $Z(x)$. In that case, the kriging estimator [see Eq. (1) ] can be written as

$$
\begin{aligned}
\hat{Z}(x) & =\mu(x)+\sum_{i=1}^{n} w_{i}(x)\left[Z\left(x_{i}\right)-\mu(x)\right] \\
& =\sum_{i=1}^{n} w_{i}(x) Z\left(x_{i}\right)+\left(1-\sum_{i=1}^{n} w_{i}(x)\right) \mu(x)
\end{aligned}
$$

Filtering the unknown local mean, we get

$$
\hat{Z}(x)=\sum_{i=1}^{n} w_{i}(x) Z\left(x_{i}\right), \quad \text { with } \quad \sum_{i=1}^{n} w_{i}(x)=1
$$

For the estimation error, we get the following:

$$
\begin{aligned}
\hat{Z}(x)-Z(x) & =(\hat{Z}(x)-\mu)-(Z(x)-\mu) \\
& =\sum_{i=1}^{n} w_{i}(x) R\left(x_{i}\right)-R(x)=\hat{R}(x)-R(x)
\end{aligned}
$$

The variance [see Eq. (A3)] is then given by the covariogram or the variogram [Eq. (A4)], respectively:

$$
\begin{aligned}
\sigma^{2}(x) & =\operatorname{Var}\{\hat{R}(x)\}+\operatorname{Var}\{R(x)\}-2 \operatorname{Cov}\{\hat{R}(x), R(x)\} \\
& =\sum_{i, j=1}^{n} w_{i}(x) w_{j}(x) C_{R}\left(x_{i}-x_{j}\right)+C_{R}(0)-2 \sum_{i}^{n} w_{i}(x) C_{R}\left(x_{i}-x\right)
\end{aligned}
$$




$$
=-\sum_{i, j=1}^{n} w_{i}(x) w_{j}(x) \gamma\left(x_{i}-x_{j}\right)+2 \sum_{i}^{n} w_{i}(x) \gamma\left(x_{i}-x\right)
$$

To minimize the error variance subject to the constraint [Eq. (6) ], we involve the Lagrange parameter $2 \lambda(x)$ and minimize

$$
L=\sigma^{2}(x)+2 \lambda(x)\left(1-\sum_{i=1}^{n} w_{i}(x)\right)
$$

Setting derivatives of the Lagrange function $L$ with respect to $w_{1}, \ldots, w_{n}, \lambda$ to zero yields the kriging weights as a linear equation system:

$$
\left(\begin{array}{cccc}
\gamma\left(x_{1}-x_{1}\right) & \cdots & \gamma\left(x_{1}-x_{n}\right) & 1 \\
\vdots & \ddots & \vdots & \vdots \\
\gamma\left(x_{n}-x_{1}\right) & \cdots & \gamma\left(x_{n}-x_{n}\right) & 1 \\
1 & \cdots & 1 & 0
\end{array}\right)\left(\begin{array}{c}
w_{1} \\
\vdots \\
w_{n} \\
\lambda
\end{array}\right)=\left(\begin{array}{c}
\gamma\left(x_{1}-x\right) \\
\vdots \\
\gamma\left(x_{n}-x\right) \\
1
\end{array}\right)
$$

From the linear equation system, the interpolation, and Eq. (A9) the error is deduced

$$
\begin{aligned}
\hat{Z}(x) & =\left(\begin{array}{c}
w_{1} \\
\vdots \\
w_{n}
\end{array}\right)^{T}\left(\begin{array}{c}
Z\left(x_{1}\right) \\
\vdots \\
Z\left(x_{n}\right)
\end{array}\right), \\
\operatorname{Var}(\hat{Z}(x)-Z(x)) & =\left(\begin{array}{c}
w_{1} \\
\vdots \\
w_{n} \\
\lambda
\end{array}\right)^{T}\left(\begin{array}{c}
\gamma\left(x_{1}, x\right) \\
\vdots \\
\gamma\left(x_{n}, x\right) \\
1
\end{array}\right)
\end{aligned}
$$

The kriging method has the following properties:

1) Kriging estimation is unbiased $E\left[\hat{Z}\left(x_{i}\right)\right]=E\left[Z\left(x_{i}\right)\right]$.

2) Kriging estimation is exact at observed values: $\hat{Z}\left(x_{i}\right)=Z\left(x_{i}\right)$ with respect to measurement errors.

3) The kriging estimation is the best linear unbiased estimator of $Z(x)$ if assumptions hold.

\section{Acknowledgments}

The work was funded by the DLR, Germany Aerospace Center project Land-based and Onboard Wake Systems (L-Bows). Computer resources for this project were provided by the Gauss Center for Supercomputing/Leibniz Supercomputing Center under grant pr63zi. We would like to thank M. Manhart for providing the original version of the large-eddy-simulation code MGLET.

\section{References}

[1] Gerz, T., Holzäpfel, F., and Darracq, D., "Commercial Aircraft Wake Vortices," Progress in Aerospace Sciences, Vol. 38, No. 3, 2002, pp. 181-208. doi:10.1016/S0376-0421(02)00004-0

[2] Gerz, T., Holzäpfel, F., Bryant, W., Köpp, F., Ferch, M., Tafferner, A., and Winckelmans, G., "Research Towards a Wake-Vortex Advisory System for Optimal Aircraft Spacing," Comptes Rendus Physique, Vol. 6, Nos. 4-5, 2005, pp. 501-523. doi:10.1016/j.crhy.2005.06.002

[3] Spalart, P. R., "Airplane Trailing Vortices," Annual Review of Fluid Mechanics, Vol. 30, No. 1, 1998, pp. 107-138. doi:10.1146/annurev.fluid.30.1.107
[4] Holzäpfel, F., and Steen, M., "Aircraft Wake-Vortex Evolution in Ground Proximity: Analysis and Parameterization," AIAA Journal, Vol. 45, No. 1, 2007, pp. 218-227. doi: $10.2514 / 1.23917$

[5] Critchley, J., and Foot, P., "UK CAA Wake Vortex Database: Analysis of Incidents Reported Between 1982 and 1990,' Civil Aviation Authority, Paper 91, Gatwick, 1991.

[6] "Challenges of Growth," Eurocontrol Summary Rept., Brussels, June 2013.

[7] "Federal Aviation Administration (FAA) Aircraft Wake Turbulence Re-Categorization (RECAT) Updates," U.S. Dept. of Transportation, Federal Aviation Administration Safety Alert for Operation 14007 Dec. 2014, http://www.faa.gov/other_visit/aviation_industry/airline operators/airline_safety/safo/all_safos/media/2014/safo14007.pdf [retrieved 2016].

[8] "Implementation of the RECAT-EU Wake Turbulence Separation Scheme At Paris Charles De Gaulle, Paris-Le Bourget and Pontoise-Cormeilles-enVexin Airports from March 22nd 2016," Service de l'Information Aéronautique SIA-2016-03, Bordeaux-Mérignac, 2016, https://www.sia.aviation-civile.gouv.fr.

[9] Breitsamter, C., "Wake Vortex Characteristics of Transport Aircraft," Progress in Aerospace Sciences, Vol. 47, No. 2, 2011, pp. 89-134. doi:10.1016/j.paerosci.2010.09.002

[10] Coustols, E., Stumpf, E., Jaquin, L., Moens, F., Vollmers, H., and Gerz, T., "Minimized Wake: A Collaborative Research Programme on Aircraft Wake Vortices," AIAA Paper 2003-0938, 2003.

[11] Stumpf, E., "Study of Four-Vortex Aircraft Wakes and Layout of Corresponding Aircraft Configurations," Journal of Aircraft, Vol. 42, No. 3, 2005, pp. 722-730. doi:10.2514/1.7806

[12] Ortega, J. M., Bristol, R. L., and Savas, Ö., "Wake Alleviation Properties of Triangular-Flapped Wings," Journal of Aircraft, Vol. 40, No. 4, 2002 , pp. 709-721. doi: $10.2514 / 2.1703$

[13] Crouch, J. D., Miller, G., and Spalart, P. R., "Active-Control System for Breakup of Airplane Trailing Vortices," AIAA Journal, Vol. 39, No. 12 , 2001, pp. 2374-2381. doi: $10.2514 / 2.1244$

[14] Fabre, D., Jacquin, L., and Loof, A., "Optimal Perturbations in a FourVortex Aircraft Wake in Counter-Rotating Configuration," Journal of Fluid Mechanics, Vol. 451, 2002, pp. 319-328. doi:10.1017/S0022112001006954

[15] Stephan, A., Holzäpfel, F., and Misaka, T., "Aircraft Wake-Vortex Decay in Ground Proximity-Physical Mechanisms and Artificial Enhancement," Journal of Aircraft, Vol. 50, No. 4, 2013, pp. 1250-1260.

[16] Marshall, J. S., Brancher, P., and Giovannini, A., "Interaction of Unequal Anti-Parallel Vortex Tubes," Journal of Fluid Mechanics, Vol. 446, 2001, pp. 229-252.

[17] Holzäpfel, F., Stephan, A., Heel, T., and Körner, S., "Enhanced Wake Vortex Decay in Ground Proximity Triggered by Plate Lines," Aircraft Engineering and Aerospace Technology: An International Journal, Vol. 88, No. 2, 2016, pp. 206-214. doi:10.1108/AEAT-02-2015-0045

[18] Wang, J. C. H., and Schlüter, J., "Near Ground Aircraft Wake Dissipation with Obstacles," AIAA Aviation, 33rd AIAA Applied Aerodynamics Conference, AIAA Paper 2015-3298, June 2015.

[19] Leifsson, L., and Koziel, S., Simulation-Driven Aerodynamic Design Using Variable-Fidelity Models, Imperial College Press, London, 2015.

[20] Matheron, G., "Principles of Geostatistics," Economic Geology, Vol. 58, No. 8, 1963, pp. 1246-1266. doi:10.2113/gsecongeo.58.8.1246

[21] Krige, D. G., "A Statistical Approach to Some Basic Mine Valuations Problems on the Witwatersrand," Journal of the Chemical, Metallurgical and Mining Society of South Africa, Vol. 5, No. 2, 1951, pp. 119-139.

[22] Robins, R. E., and Delisi, D. P., "Potential Hazard of Aircraft Wake Vortices in Ground Effect with Crosswind," Journal of Aircraft, Vol. 30, No. 2, 1993, pp. 201-206. doi: $10.2514 / 3.48266$

[23] Türk, L., Coors, D., and Jacob, D., "Behavior of Wake Vortices Near the Ground over a Large Range of Reynolds Numbers," Aerospace Science and Technology, Vol. 3, No. 2, 1999, pp. 71-81. doi:10.1016/S1270-9638(99)80031-5

[24] Harvey, J. K., and Perry, F. J., "Flowfield Produced by Trailing Vortices in the Vicinity of the Ground," AIAA Journal, Vol. 9, No. 8, 1971, pp. 1659-1660. doi: $10.2514 / 3.6415$

[25] Dufresne, L., Baumann, R., Gerz, T., Winckelmans, G., Moet, H., and Capart, R., "Large Eddy Simulation of Wake Vortex Flows at Very High 
Reynolds Numbers: A Comparison of Different Methodologies," Aircraft Wing Advanced Technology Operations TR D1.14-16, 2005.

[26] Spalart, P. R., Strelets, M. K., Travin, A. K., and Shur, M. L., "Modelling the Interaction of a Vortex Pair with the Ground," Fluid Dynamics, Vol. 36, No. 6, 2001, pp. 899-908. doi:10.1023/A:1017958425271

[27] Proctor, F. H., Hamilton, D. W., and Han, J., "Wake Vortex Transport and Decay in Ground Effect: Vortex Linking with the Ground," 38th Aerospace Sciences Meeting and Exhibit, AIAA Paper 2000-0757, 2000.

[28] Misaka, T., Holzäpfel, F., and Gerz, T., "Large-Eddy Simulation of Aircraft Wake Evolution from Roll-Up Until Vortex Decay," AIAA Journal, Vol. 53, No. 9, 2015, pp. 2646-2670. doi:10.2514/1.J053671

[29] Stephan, A., Holzäpfel, F., and Misaka, T., "Hybrid Simulation of Wake-Vortex Evolution During Landing on Flat Terrain and with Plate Line," International Journal of Heat and Fluid Flow, Vol. 49, 2014, pp. 18-27. doi:10.1016/j.ijheatfluidflow.2014.05.004

[30] Jones, D. R., Schonlau, M., and Welch, W. J., "Efficient Global Optimization of Expensive Black-Box Functions," Journal of Global Optimization, Vol. 13, No. 4, 1998, pp. 455-492. doi:10.1023/A:1008306431147

[31] Duponcheel, M., Lonfils, T., Bricteux, L., and Winckelmans, G., "Simulations of Three-Dimensional Wake Vortices in Ground Effect Using a Fourth-Order Incompressible Code," 7th National Congress on Theoretical and Applied Mechanics, Mons, 2006.

[32] Georges, L., Geuzaine, P., Duponchel, M., Bricteux, L., Lonfils, T., Winckelmans, G., and Giovannini, A., "LES of Two-Vortex System in Ground Effect with and Without Wind," Univ. Catholique de Louvain, Inst. de Mécanique des Fluides de Toulouse TR 3.1.1-3, Louvain-laNeuve, Belgium, 2005.

[33] Manhart, M., "A Zonal Grid Algorithm for DNS of Turbulent Boundary Layer," Computer and Fluids, Vol. 33, No. 3, 2004, pp. 435-461. doi:10.1016/S0045-7930(03)00061-6
[34] Meneveau, C., Lund, T. S., and Cabot, W. H., "A Lagrangian Dynamic Subgrid-Scale Model of Turbulence," Journal of Fluid Mechanics, Vol. 319, 1996, pp. 353-385. doi:10.1017/S0022112096007379

[35] Hokpunna, A., and Manhart, M., "Compact Fourth-Order Finite Volume Method for Numerical Solutions of Navier-Stokes Equations on Staggered Grids," Journal of Computational Physics, Vol. 229, No. 20, 2010, pp. 7545-7570. doi:10.1016/j.jcp.2010.05.042

[36] Hokpunna, A., "Compact Fourth-Order Scheme for Numerical Simulations of Navier-Stokes Equations," Ph.D. Thesis, Technische Univ. München, Munich, 2009.

[37] Shaw, R. H., and Schumann, U., "Large-Eddy Simulation of Turbulent Flow Above and Within a Forest," Boundary-Layer Meteorology, Vol. 61, Nos. 1-2, 1992, pp. 47-64. doi:10.1007/BF02033994

[38] Kitanidis, P. K., Introduction to Geostatistics: Application in Hydrogeology, Cambridge Univ. Press, New York., 1997.

[39] Christakos, G., "On the Problem of Permissible Covariance and Variogram Models," Water Resources Research, Vol. 20, No. 2, 1984, pp. 251-265. doi:10.1029/WR020i002p00251

[40] Cressie, N. A. C., Statistics for Spatial Data, Wiley, New York, 1993.

[41] Schrall, J., "Numerical Optimization of Plate Line Design," M.S. Thesis, Univ. of Hagen, Hagen, Germany, 2016.

[42] Chapman, D. R., "Computational Aerodynamics Development and Outlook," AIAA Journal, Vol. 17, No. 12, 1979, pp. 1293-1313. doi:10.2514/3.61311

[43] Stephan, A., Holzäpfel, F., Misaka, T., Geisler, R., and Konrath, R., "Enhancement of Aircraft Wake Vortex Decay in Ground Proximity Experiment Versus Simulation," CEAS Aeronautical Journal, 2013, pp. 1-17. doi:10.1007/s13272-013-0094

[44] Goovaerts, P., Geostatistics for Natural Resources Evaluation, Oxford Univ. Press, New York, 1997. doi:10.1007/s13272-013-0094 